\title{
Metric Dimension of Maximal Outerplanar Graphs
}

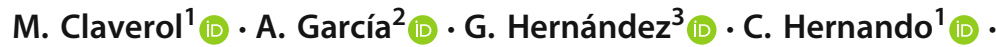 \\ M. Maureso ${ }^{1}\left(\right.$ D $\cdot$ M. Mora ${ }^{1}$ (i) $\cdot$ J. Tejel $^{2}$ (I)
}

Received: 30 October 2019 / Revised: 7 December 2020 / Accepted: 11 December 2020

(c) Malaysian Mathematical Sciences Society and Penerbit Universiti Sains Malaysia 2021

\begin{abstract}
In this paper, we study the metric dimension problem in maximal outerplanar graphs. Concretely, if $\beta(G)$ denotes the metric dimension of a maximal outerplanar graph $G$ of order $n$, we prove that $2 \leq \beta(G) \leq\left\lceil\frac{2 n}{5}\right\rceil$ and that the bounds are tight. We also provide linear algorithms to decide whether the metric dimension of $G$ is 2 and to build a resolving set $S$ of size $\left\lceil\frac{2 n}{5}\right\rceil$ for $G$. Moreover, we characterize all maximal outerplanar graphs with metric dimension 2.
\end{abstract}

Keywords Metric dimension · Resolving set · Maximal outerplanar graph

Mathematics Subject Classification 05C12 - 05C10

\section{Introduction}

Let $G=(V, E)$ be a finite connected simple graph. For two vertices $u, v \in V$, let $d(u, v)$ denote the length of a shortest path in $G$ from $u$ to $v$. If $S=\left\{x_{1}, \ldots, x_{k}\right\}$ is a set of vertices of $G$, we denote by $r(u \mid S)$ the vector of distances from $u$ to the vertices of $S$, that is, $r(u \mid S)=\left(d\left(u, x_{1}\right), \ldots, d\left(u, x_{k}\right)\right)$. We say that a vertex $x \in V$ resolves a pair of vertices $u, v \in V$ if $d(u, x) \neq d(v, x)$. A set of vertices $S \subseteq V$ is a resolving set of $G$ if every pair of distinct vertices of $G$ are resolved by some vertex in $S$. Therefore, $S$ is a resolving set if and only if $r(u \mid S) \neq r(v \mid S)$ for every pair of distinct vertices $u, v \in V(G)$. The elements of $r(u \mid S)$ are the metric coordinates of $u$ with respect to $S$. A resolving set $S$ of $G$ with minimum cardinality is a metric basis of

Communicated by Sandi Klavžar.

This project has received funding from the European Union's Horizon 2020 research and innovation programme under the Marie Skłodowska-Curie Grant Agreement No. 734922.

$凶 \quad$ M. Mora

merce.mora@upc.edu

Extended author information available on the last page of the article 
$G$. The metric dimension of $G$, denoted by $\beta(G)$, is the cardinality of a metric basis. The metric dimension problem consists of finding a metric basis.

Resolving sets in general graphs were first studied by Slater [28] and Harary and Melter [18]. Since then, computing resolving sets and the metric dimension of a graph have been widely studied in the literature due to their applications in several areas, such as network discovery and verification [2], robot navigation [23] or chemistry [6]. The reader is referred to $[1,4,5,12-17,19,25,27,33]$ and the references therein for different results and variants of the metric dimension problem of graphs.

It is well known that the metric dimension problem in general graphs is NPhard [23]. The problem remains NP-hard even when restricting to some graph classes such as bounded degree planar graphs [9]; split graphs, bipartite graphs and their complements, and line graphs of bipartite graphs [11]; and interval graphs and permutation graphs of diameter 2 [13]. Polynomial algorithms are known for trees [23]; outerplanar graphs [9]; chain graphs [12]; and $k$-edge-augmented trees, cographs and wheels [11]. A weighted variant of the metric dimension problem in several graphs, including paths, trees and cographs, can be also solved in polynomial time [11].

While the algorithms to solve the metric dimension problem in trees, wheels, or chain graphs are linear, the time complexity of the algorithm given in [9] for an outerplanar graph of order $n$ is $O\left(n^{12}\right)$. Thus, an interesting problem for such graphs is how to find more efficiently a not very large resolving set. Recall that a graph $G$ is outerplanar if it can be drawn in the plane without crossings and with all the vertices belonging to the unbounded face.

In this paper, we focus on studying the metric dimension problem in maximal outerplanar graphs. A maximal outerplanar graph, MOP graph for short, is an outerplanar graph such that the addition of an edge produces a non-outerplanar graph. In particular, given a MOP graph $G$ of order $n \geq 3$ we show that $2 \leq \beta(G) \leq\left\lceil\frac{2 n}{5}\right\rceil$ and that the bounds are tight. The lower bound is shown to be tight in Sect. 2. Moreover, all MOP graphs with metric dimension 2 are characterized. We also provide in that section a linear algorithm to decide whether the metric dimension of a MOP graph is 2. The tightness of the upper bound is shown in Sect. 3.1 by exhibiting a family of MOP graphs attaining the given bound. Section 3.2 is devoted to show that the metric dimension of a MOP graph $G$ is at most $\left\lceil\frac{2 n}{5}\right\rceil$, by building in linear time a resolving set $S$ for $G$ such that $|S|=\left\lceil\frac{2 n}{5}\right\rceil$. In [26], it is conjectured that $\beta(G) \leq\left\lceil\frac{2 n}{5}\right\rceil$ for a maximal planar graph $G$; hence, we are answering in the affirmative this conjecture for the particular case of MOP graphs. We conclude the paper with some open questions in Sect. 4. An extended abstract of this work has appeared at the 17th Spanish Meeting on Computational Geometry (EGC 2017).

To finish this section, we recall some well-known properties of MOP graphs. A MOP graph $G$ of order at least 3 is biconnected, Hamiltonian, does not contain a complete bipartite graph $K_{2,3}$ as subgraph and always admits a plane embedding such that all vertices belong to the unbounded face and every bounded face is a triangle. Unless otherwise stated, we assume throughout the paper that the MOP graph has order at least 3 and we are given this plane embedding of $G$. Thus, $G$ can be seen as a triangulation of a convex polygon. Every edge on the boundary of the unbounded face belongs to only one triangle of $G$, and any other edge (called diagonal) belongs 
to two triangles. The removal of the endvertices of a diagonal makes the graph to be disconnected. $G$ always has at least 2 vertices of degree 2, and when removing any of them (if $|G| \geq 4$ ), the resulting graph is a MOP graph. From these properties, it is straightforward to see the following result:

Remark 1 Let $G$ be a MOP graph and let $x y$ be a diagonal of $G$. If $u$ and $z$ are two vertices belonging to different components of $G \backslash\{x, y\}$, then $d(u, z)>$ $\min \{d(u, x), d(u, y)\}$.

We also recall that, from an algorithmic point of view, deciding whether a graph is planar (outerplanar) and building a (straight-line) plane embedding of a planar (outerplanar) graph can be done in linear time and space. See, for example, [7,8,22, $24,32]$ and the references therein. In the case of a MOP graph $G$, a standard technique to build in linear time and space a straight-line plane embedding such that all vertices belong to the unbounded face is the following: Remove repeatedly vertices of degree two until obtaining a triangle, draw this triangle in the plane and add the vertices of degree two in the order opposite to the order in which they were removed. Since G is a MOP graph, the vertex of degree two added in each step is always adjacent to two consecutive vertices on the boundary of the unbounded face. In particular, in linear time and space, the following properties of the embedding of the MOP graph can be built and stored: the cyclic order of the vertices along the boundary of the unbounded face, the triangular faces and their adjacencies, and, for each vertex, the cyclic order of its neighbors.

\section{MOP Graphs with Metric Dimension Two}

Given a MOP graph $G$, its metric dimension must be greater than one, as paths are the only graphs with metric dimension one (see, for example, [6]). In this section, we characterize MOP graphs with metric dimension two.

There are several papers in the literature devoted to study properties of graphs with metric dimension two and to characterize such graphs for certain families of graphs. In [30], the authors give a general characterization for a graph $G$ to have metric dimension two, based on the distance partition $\left\{U_{1}, U_{2}, \ldots, U_{k}\right\}$ of the vertices of $G$, where vertices belonging to $U_{i}$ are at distance $i$ from a distinguished vertex $v$. They also give a $O\left(n^{2} D^{4}\right)$ algorithm to check whether the metric dimension of a graph of order $n$ is two, where $D$ is the diameter of the graph. In [23], the authors show several properties that a graph with metric dimension two must satisfy.

Graphs with metric dimension two have been characterized for some families of graphs. In particular, unicyclic graphs [10] and Cayley graphs [31]. An incorrect characterization of the 2-trees with metric dimension 2 is given in [3]. Starting with a triangle, a 2-tree is formed by repeatedly adding vertices of degree 2 in such a way that each added vertex $u$ is connected to two vertices $v$ and $w$ which are already adjacent. Thus, the family of 2-trees includes MOP graphs as a subfamily.

In [3], the authors define a family $\mathcal{F}$ of 2-trees such that a 2-tree $G$ belongs to $\mathcal{F}$ if $G$ satisfies a set of twelve conditions, and they claim that a 2-tree $G$ has metric dimension 2 if and only if $G$ belongs to $\mathcal{F}$. When proving that a 2-tree $G$ with metric dimension 


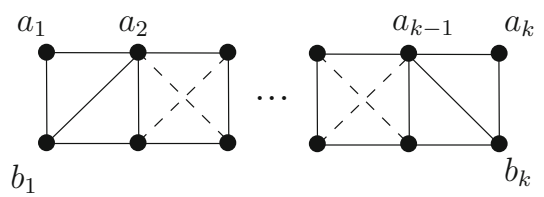

(a)

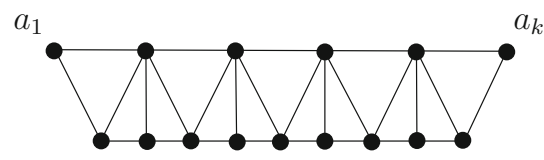

(b)

Fig. 1 a Given a 2-tree $G$ with metric dimension 2, a minimal induced 2-connected subgraph containing the basis $\left\{a_{1}, a_{k}\right\}$, as claimed in [3]. b A 2-tree with metric basis $\left\{a_{1}, a_{k}\right\}$ whose minimal induced 2-connected subgraph containing $a_{1}$ and $a_{k}$ is different from the claimed subgraph in [3]
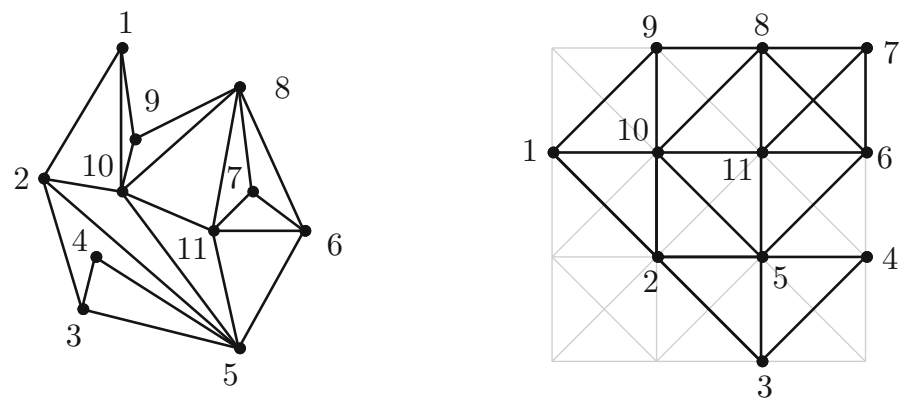

Fig. 2 Left: A graph $G$ with metric basis $S=\{1,3\}$. The metric coordinates of the vertices are: $r(1 \mid S)=$ $(0,2), r(2 \mid S)=(1,1), r(3 \mid S)=(2,0), r(4 \mid S)=(3,1), r(5 \mid S)=(2,1), r(6 \mid S)=(3,2), r(7 \mid S)=$ $(3,3), r(8 \mid S)=(2,3), r(9 \mid S)=(1,3), r(10 \mid S)=(1,2)$ and $r(11 \mid S)=(2,2)$. Right: The representation $G^{*}$ of $G$ as a subgraph of $P_{n} \otimes P_{n}$ with respect to $S$. Vertex $v$ in $G$ is mapped to vertex $v^{*}$ in $G^{*}$ such that the Cartesian coordinates of $v^{*}$ are the metric coordinates of $v$

two must belong to $\mathcal{F}$, the authors claim in one of the cases that the minimal induced 2-connected subgraph of $G$ containing the two vertices $a_{1}$ and $a_{k}$ of the basis of $G$ has the shape shown in Fig. 1a: two vertices of degree two $\left(a_{1}\right.$ and $\left.a_{k}\right)$, two vertices of degree three $\left(b_{1}\right.$ and $\left.b_{k}\right)$, a set of quadrilaterals with one of the two possible diagonals, and at most one vertex of degree five in the path $a_{1}, a_{2}, \ldots, a_{k}$. But, part (b) of Fig. 1 exhibits a 2-tree $G$ (in fact a MOP graph) with metric dimension two, $\left\{a_{1}, a_{k}\right\}$ being the only basis of $G$ and the minimal induced 2-connected subgraph of $G$ containing $a_{1}$ and $a_{k}$ is precisely $G$, contradicting the shape claimed in [3]. As a consequence, their claimed characterization cannot be used to characterize MOP graphs with metric dimension 2 .

We next give a characterization for MOP graphs with metric dimension two, based on embedding graphs with metric dimension 2 into the strong product of two paths. The strong product of two paths of order $n, P_{n} \otimes P_{n}$, has the Cartesian product $[0, n-1] \times[0, n-1]$ as set of vertices and two different vertices $(i, j)$ and $\left(i^{\prime}, j^{\prime}\right)$ are adjacent if and only if $\left|i^{\prime}-i\right| \leq 1$ and $\left|j^{\prime}-j\right| \leq 1$. The distance between two vertices of this graph is $d\left((i, j),\left(i^{\prime}, j^{\prime}\right)\right)=\max \left\{\left|i^{\prime}-i\right|,\left|j^{\prime}-j\right|\right\}$. We will consider the representation of this graph in the plane identifying vertex $(i, j)$ with the point with Cartesian coordinates $(i, j)$. In this representation, a path of length $k$ between two vertices $(i, j)$ and $\left(i^{\prime}, j^{\prime}\right)$ such that $d\left((i, j),\left(i^{\prime}, j^{\prime}\right)\right)=k$ is contained in the rectangle having $(i, j)$ and $\left(i^{\prime}, j^{\prime}\right)$ as opposite vertices and sides parallel to lines of slope 1 and -1 passing through these vertices. A set of four vertices of $P_{n} \otimes P_{n}$ of the form 

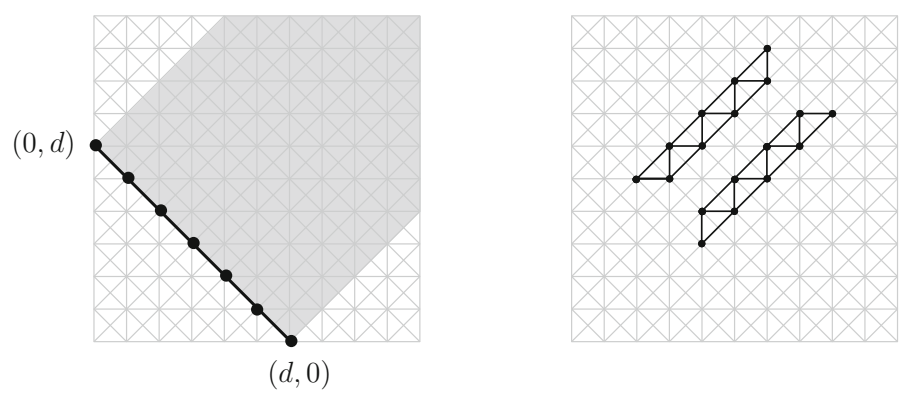

Fig. 3 Left: Illustrating Proposition 1. The shortest path from $(0, d)$ to $(d, 0)$ and the set $A_{d}$, which is in the shaded region. Right: Examples of horizontal and vertical MOP zigzags

$\{(i, j),(i, j+1),(i+1, j+1),(i+1, j)\}$, for some $i, j \in[0, D]$, is a unit square. Three vertices of $P_{n} \otimes P_{n}$ are pairwise adjacent if and only if they all belong to a unit square, and in such a case, the edges joining them form a triangle with two consecutive sides one of a unit square and the diagonal joining them.

Let $G$ be a graph with metric dimension 2 and let $S=\{u, v\}$ be a metric basis of $G$. It is straightforward to see that $G$ is isomorphic to a subgraph of the strong product $P_{n} \otimes P_{n}$. See Fig. 2 for an example. Indeed, we identify vertex $x \in V(G)$ with vertex $\left(x_{1}, x_{2}\right) \in V\left(P_{n} \otimes P_{n}\right)$, where $\left(x_{1}, x_{2}\right)=r(x \mid S)=(d(x, u), d(x, v))$. Recall that if two vertices $w_{1}$ and $w_{2}$ of $G$ are adjacent and $d\left(w_{0}, w_{1}\right)=d$ for some vertex $w_{0}$, then $d\left(w_{0}, w_{2}\right) \in\{d-1, d, d+1\}$. Thus, if $x$ and $y$ are adjacent vertices in $G$, then $|d(x, u)-d(y, u)| \leq 1$ and $|d(x, v)-d(y, v)| \leq 1$; hence, $r(x \mid S)$ and $r(y \mid S)$ are adjacent in $P_{n} \otimes P_{n}$. We denote by $G^{*}$ this representation of $G$, that is, $V\left(G^{*}\right)=\{r(x \mid S): x \in V(G)\}$ and $r(x \mid S) r(y \mid S) \in E\left(G^{*}\right)$ if and only if $x y \in E(G)$. We say that $G^{*}$ is the representation of $G$ as a subgraph of $P_{n} \otimes P_{n}$ with respect to $S$, and vertex $(i, j)$ is placed onto the point with Cartesian coordinates $(i, j)$.

For every $d \geq 1$, consider the set $A_{d}=\{(i, j) \in[0, n-1] \times[0, n-1]: i+j \geq$ $d,|j-i| \leq d\}$ (see Fig. 3 left). The following properties can be easily derived.

Proposition 1 Let $G$ be a graph with metric dimension 2, and let $S=\{u, v\}$ be a metric basis of $G$ such that $d(u, v)=d$. Consider the representation $G^{*}$ of $G$ as a subgraph of $P_{n} \otimes P_{n}$ with respect to $S$. The following properties hold:

(1) $S^{*}=\{(0, d),(d, 0)\}$ is a metric basis of $G^{*}$ and all the vertices of $G^{*}$ are in $A_{d}$.

(2) There is only one shortest $((0, d),(d, 0))$-path in $G^{*}$, and its vertices are the points $(i, j)$ such that $i+j=d$.

Proof (1) If $d=d(u, v)$, then $r(u \mid S)=(0, d)$ and $r(v \mid S)=(d, 0)$. If $x \in V(G)$, then $r(x \mid S)=\left(x_{1}, x_{2}\right)=(d(x, u), d(x, v))$. On the one hand, $x_{1}=d(x, u) \leq$ $d(x, v)+d(u, v)=d(x, v)+d=x_{2}+d$ and $x_{2}=d(x, v) \leq d(x, u)+$ $d(u, v)=d(x, u)+d=x_{1}+d$; hence, $\left|x_{1}-x_{2}\right| \leq d$. On the other hand, $x_{1}+x_{2}=d(x, u)+d(x, v) \geq d(u, v)=d$.

(2) There is only one path of length $d$ joining $(0, d)$ and $(d, 0)$ in $P_{n} \otimes P_{n}$, and its vertices are $\{(i, j): i+j=d\}$. Thus, it is also the only shortest path between $(0, d)$ and $(d, 0)$ in $G^{*}$ because we already know that $d_{G^{*}}((0, d),(d, 0))=d$. 
For $n \geq 5$, we say that a MOP graph $G$ is a MOP zigzag if $G$ has two vertices of degree 2, two vertices of degree 3 , each one of them adjacent to a different vertex of degree 2, and the rest of the vertices have degree 4. See Fig. 3 right for some examples of MOP zigzags. One can see a MOP zigzag as a MOP graph in which the diagonals form a zigzag path connecting the two vertices of degree 3 . For $n=3$, 4, we consider a triangle and a quadrilateral with a diagonal as MOP zigzags, respectively. For $n \geq 5$, it is straightforward to check that the two vertices of degree 2 form a metric basis, if $n$ is odd, and two non-adjacent vertices of degree 2 and 3 form a metric basis, if $n$ is even.

Given the representation $G^{*}$ of a graph $G$, we say that an edge $e \in E\left(G^{*}\right)$ is horizontal if $e=(i, j)(i+1, j)$, for some $i, j \geq 0$; vertical if $e=(i, j)(i, j+1)$, for some $i, j \geq 0$; 1-slope diagonal if $e=(i, j)(i+1, j+1)$, for some $i, j \geq 0$; and (-1)slope diagonal if $e=(i, j+1)(i+1, j)$, for some $i, j \geq 0$. A vertical MOP zigzag with base line a vertical edge $(i, j)(i, j+1)$ (see Fig. 3 right) is a subgraph of the strong product induced by the set of vertices $\{(i+k, j+k): 0 \leq k \leq r\} \cup\{(i+k, j+1+k)$ : $0 \leq k \leq s\}$, for some $r \geq 1$ and $s \in\{r-1, r\}$, and a horizontal MOP zigzag with base line a horizontal edge $(i, j)(i+1, j)$ is a subgraph of the strong product induced by the set of vertices $\{(i+k, j+k): 0 \leq k \leq r\} \cup\{(i+1+k, j+k): 0 \leq k \leq s\}$, for some $r \geq 1$ and $s \in\{r-1, r\}$.

For any integer $k \geq 1$, let $V_{k}=\{(i, j): i+j=k\}$. The following theorem characterizes the MOP graphs with metric dimension 2. Any of these MOP graphs consist of a base graph similar to the one shown in Fig. $4 \mathrm{c}$ and several MOP zigzags joined to this base graph (see Fig. 4d).

Theorem 1 Let $G$ be a MOP graph. Then, $\beta(G)=2$ if and only if there is a representation $G^{*}$ of $G$ as a subgraph of the strong product of two paths such that for some $d \geq 1$,

(1) $V\left(G^{*}\right) \subseteq A_{d}, V_{d} \cap A_{d} \subseteq V\left(G^{*}\right)$, and $E\left(G^{*}\right)$ contains the edges of the shortest path joining $(0, d)$ and $(d, 0)$.

(2) $V_{d+1} \cap A_{d} \subseteq V\left(G^{*}\right)$ and for each $(i, j) \in V_{d+1} \cap A_{d}, E\left(G^{*}\right)$ contains the edges $(i, j)(i-1, j)$ and $(i, j)(i, j-1)$.

(3) For every pair of vertices $(i, j+1)$ and $(i+1, j)$ of $V_{d+1}$ with $i, j \geq 1$, we have either $(i, j+1)(i+1, j) \in E\left(G^{*}\right)$ or $\{(i, j+1)(i+1, j+1),(i+1, j)(i+$ $1, j+1),(i, j)(i+1, j+1)\} \subseteq E\left(G^{*}\right)$. Moreover, if $(i, j+1)(i+1, j) \in E\left(G^{*}\right)$ belongs to two triangles of $G^{*}$, then $(i+1, j+1) \in V\left(G^{*}\right)$ and $\{(i, j+1)(i+$ $1, j+1),(i+1, j)(i+1, j+1)\} \subseteq E\left(G^{*}\right)$.

(4) Any other vertex or edge of the graph belongs to a vertical or horizontal MOP zigzag with base line the edge $(0, d)(1, d)$, or the edge $(d, 0)(d, 1)$, or any other edge of $G$ from those described in the preceding items with an endpoint in $V_{d+1}$ and the other in $V_{d+2}$, with the additional condition that two distinct maximal vertical or horizontal MOP zigzags do not share any edge.

Proof Let us see first that if a MOP graph has metric dimension 2, then it satisfies items (1)-(4). Item (1) is a consequence of Proposition 1 (see Fig. 4a).

Let us prove now (2). Recall that every edge of a MOP graph belongs to at least one triangle. Let $(i, j-1)(i-1, j)$ be an edge of the $((0, d),(d, 0))$-path (and thus, 


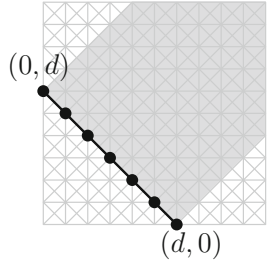

(a)

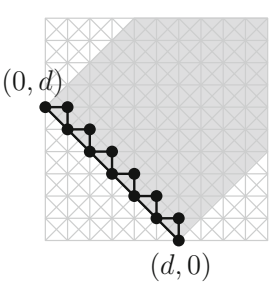

(b)

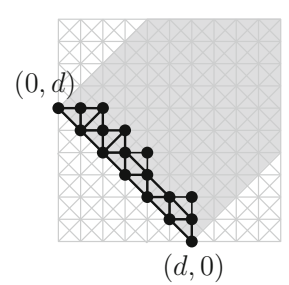

(c)

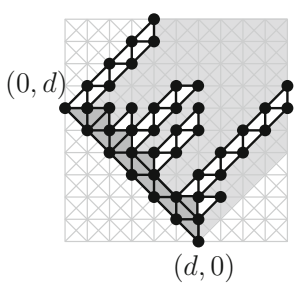

(d)

Fig. 4 An example of a MOP graph $G$ with metric dimension 2. If the vertices of a basis are at distance $d$, then $G$ can be represented as a subgraph $G^{*}$ of the strong product $P_{n} \otimes P_{n}$ such that all vertices of $G^{*}$ belong to the shaded region. Vertices described in Theorem $1(1),(2),(3)$ and (4) are added in $(a),(b),(c)$ and $(d)$, respectively. Observe that all vertices of $G^{*}$ belong to the unbounded face

$i+j-1=d)$. The only triangle of the strong product with vertices in $A_{d}$ containing this edge is that with vertices $(i, j-1),(i-1, j)$ and $(i, j)$. From here, the second item follows (see Fig. 4b).

To prove item (3), take $(i, j) \in V_{d}$. By item (2), we know that $(i, j)(i, j+1)$ and $(i, j)(i+1, j)$ are edges of $G^{*}$. Notice that the edges of the shortest $((0, d),(d, 0))$ path belong to exactly one triangle of $G^{*}$; thus, any other edge incident to $(i, j) \in V_{d}$ belongs to two triangles of $G^{*}$. Therefore, the edges $(i, j)(i, j+1)$ and $(i, j)(i+1, j)$ belong to two triangles of $G^{*}$, and there are only two possibilities, either $(i, j+1)(i+$ $1, j) \in E\left(G^{*}\right)$ or $\{(i, j+1)(i+1, j+1),(i+1, j)(i+1, j+1),(i, j)(i+1, j+1)\} \subseteq$ $E\left(G^{*}\right)$. In addition, if $(i, j+1)(i+1, j) \in E\left(G^{*}\right)$ belongs to two triangles, the only possibility is that $(i+1, j+1) \in V\left(G^{*}\right)$ and $\{(i, j+1)(i+1, j+1),(i+1, j)(i+$ $1, j+1)\} \subseteq E\left(G^{*}\right)$ (see Fig. $\left.4 \mathrm{c}\right)$.

Finally, let us prove item (4). Let $t$ be the number of triangles of the MOP graph $G$. The vertices and edges described in the preceding items (1), (2) and (3) induce a MOP graph, $G_{0}^{*}$, with $t_{0}$ triangles.

If $t=t_{0}$, then $G_{0}^{*}=G^{*}$ and we are done. Suppose now that $t>t_{0}$. In such a case, one of the edges of $G_{0}^{*}$ limiting only one triangle in $G_{0}^{*}$ must belong to two triangles in $G^{*}$. Let $e_{0}=x y$ be one of these edges, and let $z$ be the third vertex of the triangle in $G_{0}^{*}$ containing the endpoints of $e_{0}$. By definition of $G_{0}^{*}, e_{0}$ must be a horizontal edge or a vertical edge. Besides, $(0, d),(d, 0)$ and $z$ belong to the same component in $G^{*}-\{x, y\}$. Assume that $e_{0}=(i, j)(i+1, j)$ if $e_{0}$ is a horizontal edge, and $e_{0}=(i, j)(i, j+1)$ if $e_{0}$ is a vertical edge, with $i, j \geq 0$. By Remark 1 , we have that the third vertex of the other triangle of $G^{*}$ limited by $e_{0}$ must be $(i+1, j+1)$.

Let $G_{1}^{*}$ be the graph obtained by adding to the graph $G_{0}^{*}$ the vertex $(i+1, j+1)$ and the edges joining $(i+1, j+1)$ with the endpoints of $e_{0}$. Notice that one of the edges added to $G_{0}^{*}$ is a 1-slope diagonal edge, and the other one is a horizontal edge if $e_{0}$ is vertical, or a vertical edge if $e_{0}$ is horizontal.

Now, if $G^{*}=G_{1}^{*}$, we are done. Otherwise, there is an edge $e_{1}$ belonging to exactly one triangle in $G_{1}^{*}$ and to two triangles in $G^{*}$. By Remark 1, there is no 1-slope diagonal edge $(i, j)(i+1, j+1)$, with $i+j \geq d+1$, limiting two triangles in $G^{*}$. Hence, $e_{1}$ must be a horizontal edge or a vertical edge and we proceed as for $e_{0}$. We repeat this procedure until we have added $t-t_{0}$ triangles to $G_{0}^{*}$. 


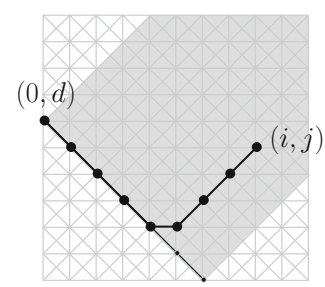

$(d, 0)$

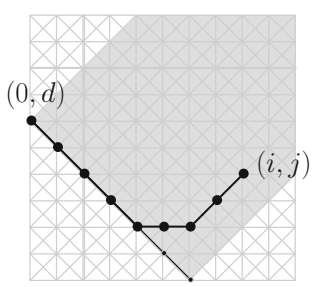

$(d, 0)$

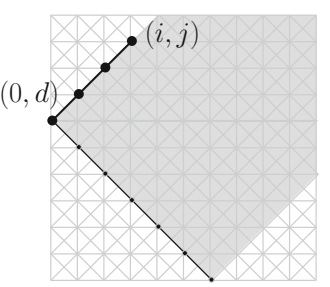

$(d, 0)$

Fig. $5 \mathrm{~A}(0, d)-(i, j)$ path of length $i$ when $i-j$ and $d$ have distinct parity (left); when $i-j$ and $d$ have the same parity and $i-j \neq-d$ (center) and when $i-j=-d$ (right)

Observe that the new triangles added to $G_{0}^{*}$ form a vertical or horizontal MOP zigzag with one of the considered base lines, since the triangles recursively added to $G_{0}^{*}$ share vertical or horizontal edges. Finally, it is not possible that two maximal vertical or horizontal MOP zigzags share an edge $e$. Indeed, in such a case, the edge $e$ should be a 1 -slope diagonal edge $e=(i, j)(i+1, j+1)$, with $i+j \geq d+1$, and $G^{*}-\{(i, j),(i+1, j+1)\}$ would be connected, a contradiction because $e$ is not an edge of the unbounded face (see Fig. 4d).

Now, we are going to prove that every graph satisfying (1) to (4) is a MOP graph with metric dimension 2. By construction, a graph satisfying conditions (1)-(4) is a biconnected plane graph with all vertices belonging to the unbounded face and any other face is a triangle. Therefore, $G$ is a MOP graph. Moreover, $d((0, d),(i, j))=i$ and $d((d, 0),(i, j))=j$. Indeed, it is easy to give a path of length $i$ from $(0, d)$ to $(i, j)$ using some vertices of the shortest $(0, d)-(d, 0)$ path; all the vertices $\left(i^{\prime}, j^{\prime}\right)$ such that $i^{\prime} \leq i, j^{\prime} \leq j$ and $i^{\prime}-j^{\prime}=i-j$; and vertex $((d+i-j) / 2,1+(d-(i-j)) / 2) \in V_{d+1}$, whenever $d$ and $i-j$ have the same parity and with $i-j \neq-d$ (see Fig. 5). In a similar way, a path of length $j$ from $(d, 0)$ to $(i, j)$ can be given. Thus, $\{(0, d),(d, 0)\}$ is a resolving set. Since $G$ is not a path, we have $\beta(G)=\beta\left(G^{*}\right)=2$.

If $G$ is a MOP graph with metric dimension 2, we denote by $G_{0}^{*}$ the graph induced by the vertices and edges described in items (1)-(3) of Theorem 1.

Deciding whether the metric dimension of a MOP graph is 2 can be done in linear time, as the following theorem shows.

Theorem 2 Given a MOP graph $G$ of order $n$, we can decide in linear time and space whether the metric dimension of $G$ is 2 .

Proof We first recall that (straight-line) plane embeddings of MOP graphs such that all vertices belong to the unbounded face can be found in linear time and space, so we may assume that the cyclic order of the vertices of $G$ along the boundary of the unbounded face is known, as well as faces, and adjacency lists in which the neighbors of each vertex are sorted.

The statement is obvious for $n \leq 5$, since all MOPs of order at most 5 have metric dimension 2. From now on, suppose that $n \geq 6$.

From Theorem 1, the representation of a MOP graph $G$ with metric dimension 2 consists of the graph $G_{0}^{*}$ together with some vertical and horizontal MOP zigzags joined to $G_{0}^{*}$. Note that every vertical or horizontal MOP zigzag finishes in a vertex of 

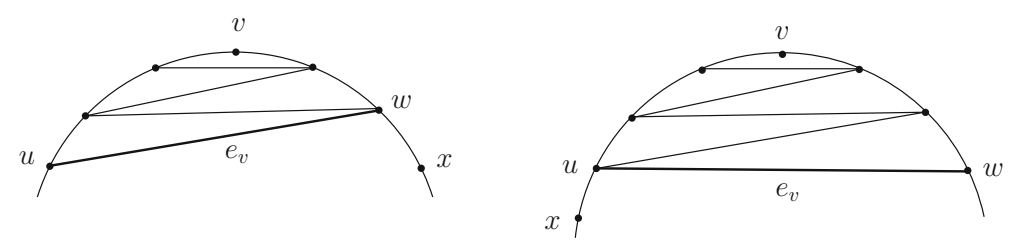

Fig. 6 Examples of maximal MOP zigzags around $v$. On the left, vertices $x$ and $u$ are non-adjacent, and on the right, vertices $x$ and $w$ are non-adjacent

degree 2 in $G$. Hence, in the first step of the algorithm we calculate the maximal MOP zigzag around $v$ for every vertex $v$ of degree 2, denoted by $G_{v}$. The set of vertices of $G_{v}$ is the maximal set of consecutive vertices $S_{v}=\{u, \ldots, v, \ldots, w\}$ of $G$ around $v$ such that the subgraph induced by $S_{v}$ is a MOP zigzag. This subgraph can be calculated by alternately exploring the vertices preceding and following $v$ (see Fig. 6). Note that by definition $u w$ is an edge of $G_{v}$ such that one of its endvertices has degree 2 in $G_{v}$ and the other one has degree 3 if $G_{v}$ is not a triangle. We call this edge the base edge of $G_{v}$ and will be denoted by $e_{v}$. Since the complexity of computing $G_{v}$ depends only on its size $d_{v}$, computing $G_{v}$ requires $O\left(d_{v}\right)$ time and space.

If $d_{v}=n$ for some vertex $v$ of degree 2 , then $G$ is a MOP zigzag and its metric dimension is 2 . Thus, we may assume that $G$ is not a MOP zigzag and we may also assume that the vertices of $G$ are clockwise ordered along its boundary. The cyclic order of the vertices of degree 2 along the boundary of $G$ implies a cyclic order on the maximal MOP zigzags that we will call their natural order. According to this natural order, we can store in a cyclic ordered list $L$ the base edges $e_{v}$. This can be done in linear time and space.

Observe that if the base edge $e_{v}$ of a maximal MOP zigzag $G_{v}$ belongs to another maximal MOP zigzag, then the union of these two maximal MOP zigzags is $G$, because maximal MOP zigzags consist of consecutive vertices around the vertices of degree 2. This implies that $G$ has only two vertices of degree 2, and since $G$ is not a MOP zigzag by assumption, $G$ must be a MOP zigzag plus a vertex of degree 3 (see Fig. 7a for an example). In this case, checking whether $G$ has metric dimension 2 is done in linear time, since by Theorem 1 only vertices of degree 2 or 3 can belong to a metric basis. Thus, we may assume that $G$ is neither a MOP zigzag nor a MOP zigzag plus a vertex of degree 3. Hence, two base edges can share at most one endpoint.

Since the base edges are diagonals of the MOP, they separate all maximal MOP zigzags (see Fig. 7b for an example), implying that two maximal MOP zigzags are disjoint or have exactly one common vertex, being this last case possible only if the MOP zigzags are consecutive in the natural order and the common vertex is an endpoint of both base edges. We can also store in linear time and space the sizes of the maximal MOP zigzags and their (possible) common vertices.

Since two maximal MOP zigzags share at most one vertex, the sum of the sizes of all maximal MOP zigzags, $d_{v}+d_{v^{\prime}}+\cdots$, is at most $2 n$. Then, the overall complexity of computing all maximal MOP zigzags is $O\left(d_{v}\right)+O\left(d_{v^{\prime}}\right)+\cdots=c_{v} d_{v}+c_{v^{\prime}} d_{v^{\prime}}+\cdots \leq$ $c\left(d_{v}+d_{v^{\prime}}+\cdots\right) \leq c 2 n$, where $c_{v}, c_{v^{\prime}}, \ldots$ are constants and $c$ is the maximum of 


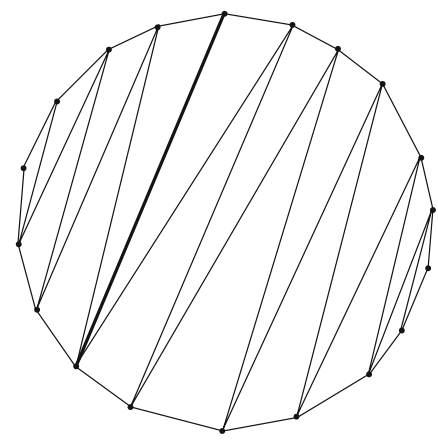

(a)

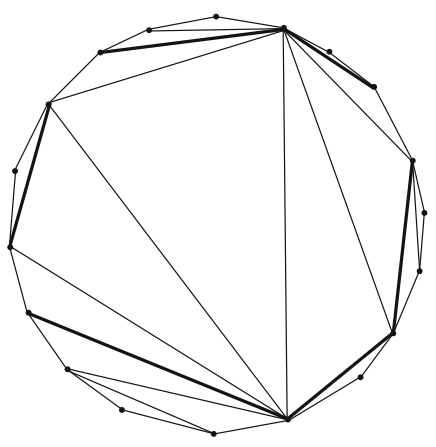

(b)

Fig. 7 a A MOP zigzag plus a vertex of degree three. The two maximal MOP zigzags have the same base edge (thick edge). b A MOP graph and its maximal MOP zigzags. Thick edges are the base edges of the maximal MOP zigzags

these constants. Therefore, this first step of computing all maximal MOP zigzags only requires linear time and space.

In the second step of the algorithm, we check whether there exists a pair of vertices defining a metric basis. Before explaining this step, we make some observations.

Suppose that $G$ has metric dimension 2 with metric basis $\{x, y\}$, and consider the graph $G_{0}^{*}$ for this basis.

First, notice that if $v$ is a vertex of degree 2 not belonging to $\{x, y\}$, then the maximal MOP zigzag $G_{v}$ does not always coincide with a horizontal or vertical MOP zigzag of those described in the embedding of Theorem 1, because the base edge $e_{v}$ of $G_{v}$ can be a $(-1)$-slope diagonal edge of $G_{0}^{*}$. This case (see, for example, the fourth MOP zigzag when moving along the border of $G_{0}^{*}$ from $(0, d)$ in Fig. $\left.4 \mathrm{~d}\right)$ only happens when the triangle defined by the vertices $(i, j+1),(i+1, j)$ and $(i+1, j+1)$, with $(i, j+1),(i+1, j) \in V_{d+1}$, belongs to $G_{0}^{*}$ and $G_{v}$ at the same time. Besides, all maximal MOP zigzags (except the ones defined by the vertices in the metric basis, if any of these vertices has degree 2 ) must be connected to $G_{0}^{*}$ precisely in their natural order.

Second, also notice that if a maximal MOP zigzag $G_{v}$ is connected to $G_{0}^{*}$ using a horizontal edge $(i, j)(i+1, j)$ or using a vertical edge $(i, j)(i, j+1)$ as base edge, then $(i, j)$ must be an endpoint of $e_{v}$ of degree 2 in $G_{v}$ (see Fig. 3 right). When $G_{v}$ is different from a triangle and is connected to $G_{0}^{*}$ using a $(-1)$-slope diagonal edge $(i, j)(i+1, j-1)$ as base edge, then either $(i, j)$ or $(i+1, j-1)$ is the vertex of degree 2 of the base edge $e_{v}$, depending on whether $G_{v}$ contains the horizontal edge $(i+1, j)(i+2, j)$ (see, for example, the fourth MOP zigzag in Fig. 4d) or contains the vertical edge $(i+1, j)(i+1, j+1)$, respectively. Therefore, given an edge $e$ on the boundary of $G_{0}^{*}$, deciding whether a maximal MOP zigzag $G_{v}$ can be connected to $G_{0}^{*}$ using $e$ (or the diagonal edge incident to it) only requires constant time checking $e_{v}$ and the degree of its vertices in $G_{v}$.

Lastly, two maximal MOP zigzags cannot be connected to two consecutive edges on the boundary of $G_{0}^{*}$ if the first edge is vertical and the second one is horizontal, because 
otherwise they would share at least one edge, contradicting item (4) of Theorem 1. For the same reason, if two maximal MOP zigzags are connected to two consecutive edges on the boundary of $G_{0}^{*}$, the first one horizontal and the second one vertical, then at least one of these zigzags is a triangle (see, for example, the second and third MOP zigzags in Fig. 4d). As a consequence, deciding whether two maximal MOP zigzags can be connected consecutively to $G_{0}^{*}$ can also be done in constant time.

With all these observations, we now describe the second step of the algorithm. Let $S=\left\{u_{1}, u_{2}, \ldots, u_{k}\right\}$ be the set of vertices of degree 2 or 3 of $G$, clockwise ordered when moving along the boundary of $G$. From Theorem 1, we deduce that, if $G$ has metric dimension 2, then a metric basis of $G$ is formed by two consecutive vertices $u_{i}$ and $u_{i+1}$ in $S$ (where $u_{k+1}=u_{1}$ ). Thus, in the second step of the algorithm, we check whether the set $\left\{u_{i}, u_{i+1}\right\}$ is a metric basis of $G$, for every $i \in\{1, \ldots, k\}$.

Given a pair $\left\{u_{i}, u_{i+1}\right\}$, this can be done as follows. Suppose that there are $d_{i}-1$ vertices between $u_{i}$ and $u_{i+1}$ when traveling clockwise along the boundary of $G$. Note that using these vertices, checking (and building) whether a graph $G_{0}^{*}$ as described in items (1), (2) and (3) of Theorem 1 exists can be done in $O\left(d_{i}\right)$ time and space. If $G_{0}^{*}$ does not exist, we continue with the following pair $\left\{u_{i+1}, u_{i+2}\right\}$.

If such a graph $G_{0}^{*}$ exists and $\left\{u_{i}, u_{i+1}\right\}$ is a metric basis, the rest of the vertices of $G$ must belong to maximal MOP zigzags joined to $G_{0}^{*}$ in their natural order. Note that, if a vertex of $\left\{u_{i}, u_{i+1}\right\}$ has degree 2 , then its maximal MOP zigzag is included in $G_{0}^{*}$. Checking whether the rest of maximal MOP zigzags can be joined to $G_{0}^{*}$ is done in $O\left(d_{i}\right)$ time, by simultaneously visiting the edges on the boundary of $G_{0}^{*}$ (clockwise) and exploring the cyclic ordered list $L$. The first active edge is the one after $u_{i+1}$ along the boundary of $G_{0}^{*}$, clockwise, and the first active base edge is the base edge of the first maximal MOP zigzag with endpoints after $u_{i+1}$ in the natural order. If the active base edge is neither the active edge nor a $(-1)$-slope diagonal edge of $G_{0}^{*}$ incident to the active edge of $G_{0}^{*}$, then we go through the following edge of $G_{0}^{*}$ that will be the new active edge of $G_{0}^{*}$. When the active base edge coincides with the active edge of $G_{0}^{*}$ or with the (-1)-slope diagonal of the triangle of $G_{0}^{*}$ to which the active edge belongs, we check whether the corresponding active maximal MOP zigzag can be connected to $G_{0}^{*}$ satisfying the former analyzed restrictions of a MOP of metric dimension 2: A vertex of degree 2 of the active base edge matches with the type of the active edge, and there is no conflict with the last maximal MOP zigzag previously connected to $G_{0}^{*}$. If it is not possible to connect the active maximal MOP zigzag to $G_{0}^{*}$, we stop the exploration because $\left\{u_{i}, u_{i+1}\right\}$ cannot be a metric basis. Otherwise, we join the maximal MOP zigzag to $G_{0}^{*}$ and continue the exploration with the following edge on the boundary of $G_{0}^{*}$ (or the following one if the maximal MOP zigzag has been joined using a diagonal edge) and the following base edge in $L$ that become the active edges.

After visiting all the edges of the boundary of $G_{0}^{*}$, if all maximal MOP zigzags (different from the ones corresponding to $u_{i}$ and $u_{i+1}$ whenever these vertices have degree 2) have been joined to $G_{0}^{*}$ and the resulting graph contains all the vertices of $G$, then $G$ has metric dimension 2. Otherwise, $\left\{u_{i}, u_{i+1}\right\}$ is not a metric basis and we proceed with the following pair of vertices $\left\{u_{i+1}, u_{i+2}\right\}$. Recall that, during the exploration, deciding whether a maximal MOP zigzag can be connected to $G_{0}^{*}$ or whether two maximal MOP zigzags can be connected consecutively only requires constant time. Moreover, at each step, the number of vertices added to $G_{0}^{*}$ can be 
Fig. 8 Fan $F_{1,6}$. Black vertices form a metric basis $S$. For a vertex not in $S$, its metric coordinates are given

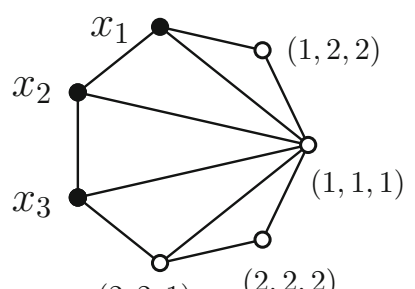

$(2,2,1)$

updated in constant time since the sizes of the maximal MOP zigzags have been stored. This number is the size of the joined maximal MOP zigzag minus the number of common vertices with $G_{0}^{*}$, that is, 2 if the base edge matches with a horizontal or vertical edge and 3 if it matches with a diagonal edge. Thus, checking whether $\left\{u_{i}, u_{i+1}\right\}$ is a metric basis is done in $O\left(d_{i}\right)$ time. Therefore, the complexity of checking for all possible pairs if they form a metric basis is $\sum O\left(d_{i}\right)$, and since $\sum d_{i}=n$, this second step also requires linear time and space.

\section{Upper Bound on the Metric Dimension of MOP Graphs}

In this section, we show that $\beta(G) \leq\left\lceil\frac{2 n}{5}\right\rceil$ for any MOP graph $G$ of order $n$. We also show that, for some special MOP graphs of order $n$, their metric dimension is $\left\lceil\frac{2(n-2)}{5}\right\rceil$. Hence, the upper bound $\left\lceil\frac{2 n}{5}\right\rceil$ is tight when $n$ is a multiple of 5 .

In the figures, we will assume that the vertices of a MOP graph $G$ are placed on a circle labeled clockwise from 1 to $n$. The edges will be drawn on or inside the circle as segments or arcs.

\subsection{Fan Graphs}

We first study the metric dimension of a special family of MOP graphs, the fan graphs. A fan graph of order $n$, denoted by $F_{1, n-1}$, is a MOP graph such that one of the vertices is connected to the $n-1$ remaining vertices. Fans are closely related to wheels. A wheel graph of order $n$ is obtained by adding a new vertex adjacent to all vertices of a cycle of order $n-1$. Thus, a fan of order $n$ can be obtained from a wheel of order $n$ by deleting an edge not incident to the vertex of degree $n-1$. It is known that wheels of order $n$ have metric dimension equal to $\left\lceil\frac{2 n}{5}\right\rceil$ [27]. We prove in this section a close result for fans, concretely, that the metric dimension of a fan of order $n$ is $\left\lceil\frac{2(n-2)}{5}\right\rceil$. For $n=3,4,5,6$, one can easily verify that $\beta\left(F_{1, n-1}\right)=2$. For $n=7$, we have $\beta\left(F_{1,6}\right) \geq 3$. This result follows from the fact that $n \leq \beta+D^{\beta}$ for a graph with metric dimension $\beta$ and diameter $D$ (see [23]). As $F_{1,6}$ has diameter 2 , if it had metric dimension 2 , then $n$ would be at most 6 . In addition, the three black vertices of Fig. 8 form a metric basis for $F_{1,6}$, so $\beta\left(F_{1,6}\right)=3$.

In the following theorem, we prove that $\beta\left(F_{1, n-1}\right)=\left\lceil\frac{2(n-2)}{5}\right\rceil$, for $n \geq 8$. The proof is based on locating-dominating sets. Given a graph $G=(V, E)$, let $N(u)$ be the set of neighbors of $u$ in $G$, that is, $N(u)=\{v: u v \in E(G)\}$. A set $S \subseteq V$ is a 

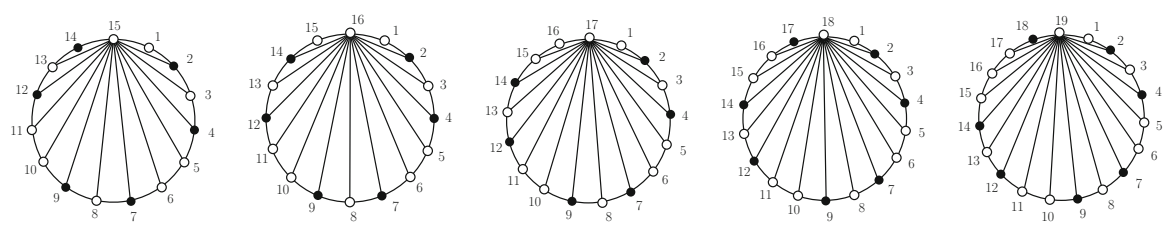

Fig. 9 The set of black vertices is a metric basis of the fans of order 15, 16, 17, 18 and 19

dominating set if every vertex not in $S$ is adjacent to some vertex in $S$. A set $S \subseteq V$ is a locating-dominating set, if $S$ is a dominating set and $N(u) \cap S \neq N(v) \cap S$ for every two different vertices $u$ and $v$ not in $S$. The location-domination number of $G$, denoted by $\lambda(G)$, is the minimum cardinality of a locating-dominating set. It is easy to show that any locating-dominating set is a resolving set. Thus, $\beta(G) \leq \lambda(G)$.

Theorem 3 Let $n \geq 8$. Then,

$$
\beta\left(F_{1, n-1}\right)=\left\lceil\frac{2(n-2)}{5}\right\rceil .
$$

Proof Observe that $\beta\left(F_{1, n-1}\right) \geq 3$, because $F_{1, n-1}$ is not a path and graphs with metric dimension 2 and diameter 2 have order at most 6 . Suppose that the vertices of $F_{1, n-1}$ are labeled so that $n$ is the vertex of degree $n-1$, and let $P$ be the path of order $n-1$ induced by vertices from 1 to $n-1$.

We first prove that $\beta\left(F_{1, n-1}\right) \leq\left\lceil\frac{2(n-2)}{5}\right\rceil$. In [29], it is shown that a path of order $n-2$ has a locating-dominating set of size $\left\lceil\frac{2(n-2)}{5}\right\rceil$ such that at least one endpoint of the path does not belong to it. Using this fact, we derive that the path of order $n-2$ induced by the vertices from 2 to $n-1$ has a locating-dominating set $S$ of size $\left\lceil\frac{2(n-2)}{5}\right\rceil$ such that $2 \notin S$. We claim that $S$ is a resolving set for $F_{1, n-1}$. On the one hand, as $n \geq 8$, then $|S| \geq 3$, so $n$ is the only vertex at distance 1 from every vertex of $S$. On the other hand, 1 is the only vertex at distance 2 from every vertex of $S$, because of the choice of $S$. Finally, every other vertex has a different vector of distances to $S$ because their neighborhoods in $S$ are different, so that the 1's in the vectors of distances to $S$ are located in different places. Consequently, $S$ is a resolving set of $F_{1, n-1}$, and hence, $\beta\left(F_{1, n-1}\right) \leq\left\lceil\frac{2(n-2)}{5}\right\rceil$.

We now prove that $\beta\left(F_{1, n-1}\right) \geq\left\lceil\frac{2(n-2)}{5}\right\rceil$. Let $S$ be a metric basis of $F_{1, n-1}$. Since $d(i, n)=1$ for $1 \leq i \leq n-1$, vertex $n$ belongs to $S$ only if it has the same coordinates as another vertex $i$ with respect to the set $S \backslash\{n\}$. Then, $(S \backslash\{n\}) \cup\{i\}$ is also a metric basis of $F_{1, n-1}$. Hence, we may assume that $n \notin S$ and $n$ is the only vertex with all metric coordinates 1 , because $\beta\left(F_{1, n-1}\right) \geq 3$. Since $F_{1, n-1}$ has diameter 2, all metric coordinates of vertices not in $S$ are 1 or 2 . There is at most one vertex with all metric coordinates 2 . If there is no vertex with all metric coordinates 2 , then $S$ is also a locating-dominating set of the path $P$ of order $n-1$. Hence, $\beta\left(F_{1, n-1}\right) \geq \lambda\left(P_{n-1}\right)=\left\lceil\frac{2(n-1)}{5}\right\rceil \geq\left\lceil\frac{2(n-2)}{5}\right\rceil$. If there is one vertex $i_{0}$ with all metric coordinates 2 , then $S$ must be a locating-dominating set for $P-i_{0}$. If $i_{0} \in\{1, n-1\}$, then $P-i_{o}$ is a path of order $n-2$ and $\beta\left(F_{1, n-1}\right) \geq \lambda\left(P_{n-2}\right)=\left\lceil\frac{2(n-2)}{5}\right\rceil$. If $i_{0} \in\{3, \ldots, n-3\}$, then $P-i_{0}$ has two connected components that are paths of order 
Fig. 10 Vertices $i$ and $j$ are

resolved either by $i-1$ or $j+1$

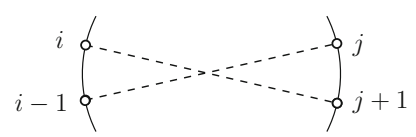

$r=i_{0}-1$ and $s=n-1-i_{0}$, respectively, with $r+s=n-2$, and we have

$$
\beta\left(F_{1, n-1}\right) \geq \lambda\left(P_{r}\right)+\lambda\left(P_{s}\right)=\left\lceil\frac{2 r}{5}\right\rceil+\left\lceil\frac{2 s}{5}\right\rceil \geq\left\lceil\frac{2(n-2)}{5}\right\rceil .
$$

Finally, if $i_{0}=2$, then $1 \notin S$ and 1 would also be at distance 2 from every vertex in $S$, a contradiction. Therefore, $i_{0} \neq 2$, and analogously, $i_{0} \neq n-2$.

It can be easily verified that if $n$ is $5 k, 5 k+1$ or $5 k+2$ for some $k$, then $S=$ $\{2+5 r: 0 \leq r<\lfloor n / 5\rfloor\} \cup\{4+5 r: 0 \leq r<\lfloor n / 5\rfloor\}$ is a metric basis of $F_{1, n-1}$, and if $n$ is $5 k+3$ or $5 k+4$ for some $k$, then $S=\{2+5 r: 0 \leq r<\lfloor n / 5\rfloor\} \cup\{4+5 r$ : $0 \leq r<\lfloor n / 5\rfloor\} \cup\{n-1\}$ is a metric basis of $F_{1, n-1}$ (see Fig. 9).

\subsection{Upper Bound}

The main goal of this section is to show that every MOP graph $G=(V, E)$ of order $n$ has a resolving set $S$ of size $\left\lceil\frac{2 n}{5}\right\rceil$ that can be built in linear time. For this purpose, we will begin with a certain set $S$ of vertices of size $\left\lceil\frac{2 n}{5}\right\rceil$. If $S$ is a resolving set, we are done. Otherwise, we will describe how $S$ can be modified to obtain a resolving set of the same size. We will refer to the vertices belonging to $S$ as black vertices and vertices not in $S$ as white vertices. Recall that the vertices of $G$ are placed on a circle and labeled clockwise from 1 to $n$, so that all the edges are drawn inside the circle. A run will be a maximal set of consecutive vertices of the same color along the circle. We will denote by $[i, j]$ the set of vertices $\{i, i+1, \ldots, j-1, j\}$, if $i<j$, and the set $\{i, i+1, \ldots, n, 1, \ldots, j-1, j\}$, if $i>j$.

We next prove some technical results.

Lemma 1 Let $G$ be a MOP graph of order $n$ and $i, j \in[1, n]$. If $i, j, i-1$ and $j+1$ are four different vertices, then $i$ and $j$ are resolved by either $i-1$ or $j+1(\bmod n)$.

Proof Observe that $G$ cannot contain at the same time the edges $(i, j+1)$ and $(j, i-1)$ because they cross, whenever $j+1 \neq i-1(\bmod n)$. Then, either $i-1$ or $j+1$ resolves $i$ and $j$. See Fig. 10.

We have seen in Sect. 3.1 that a resolving set of the fan can be obtained with alternating white runs of size 1 and 2 separated by black runs of size 1 . Such a set is not a resolving set for a general MOP graph $G$; however, these kinds of sets will play an important role to construct a resolving set of $G$. This leads us to the following definition.

We say that an interval $[i, j]$ is $(1,2)$-alternating if and only if all its white runs have size one or two, black runs have size one and there are no consecutive white runs of the same size. 


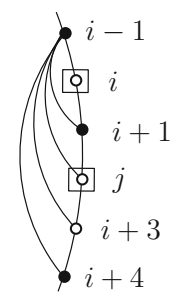

(a)

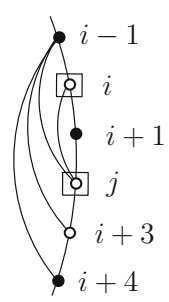

(b)

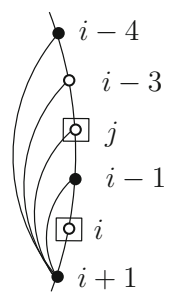

(c)

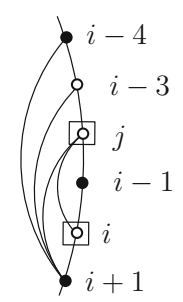

(d)

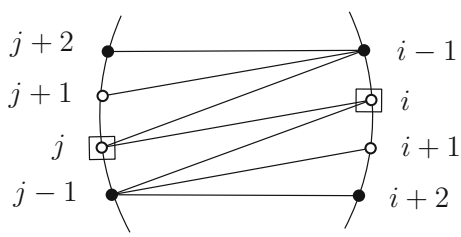

(e)

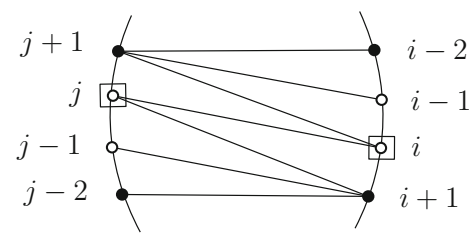

(g)

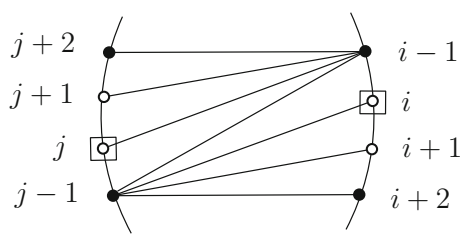

(f)

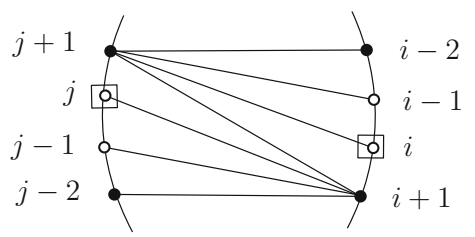

(h)

Fig. 11 Eight cases in which two white vertices $i$ and $j$, squared in the figure, are not resolved by black vertices

The next lemma shows when two white vertices of a $(1,2)$-alternating interval are not resolved by any black vertex of the interval.

Lemma 2 Let $G$ be a MOP graph and let $\left[i_{1}, i_{2}\right]$ be a $(1,2)$-alternating interval such that the first and last vertices, $i_{1}$ and $i_{2}$, are black. Let $S^{\prime}$ be the set of black vertices in the interval. The following properties hold.

(1) Let $i \in\left[i_{1}, i_{2}\right]$ belong to a white run of size 1. Then, $r\left(i \mid S^{\prime}\right)=r\left(j \mid S^{\prime}\right)$ for some $j \in\left[i_{1}, i_{2}\right]$ if and only if $j$ belongs to a white run of size 2 and one of the four cases (a), (b), (c) or (d) of Fig. 11 holds.

(2) Let $i, j \in\left[i_{1}, i_{2}\right]$ belong to white runs of size 2. Then, $r\left(i \mid S^{\prime}\right)=r\left(j \mid S^{\prime}\right)$ if and only if one of the four cases $(e),(f),(g)$, or (h) of Fig. 11 holds.

(3) If $i \in\left[i_{1}, i_{2}\right]$ is a white vertex, then there is at most one white vertex $j \in\left[i_{1}, i_{2}\right]$ such that $r\left(i \mid S^{\prime}\right)=r\left(j \mid S^{\prime}\right)$.

Proof Let us prove item (1). By Lemma 1, two white vertices belonging to two different runs of size 1 are always resolved by vertices in $S^{\prime}$. Suppose now that $i$ belongs to a white run of size 1 and $j$ belongs to a white run of size 2. If $r\left(i \mid S^{\prime}\right)=r\left(j \mid S^{\prime}\right)$, then, again by Lemma 1 , either $j$ is $i+2$ and $j$ is connected to $i-1$, or $j$ is $i-2$ and $j$ is connected to $i+1$ (see Fig. 11 top). Suppose first that $j=i+2$ and $j$ is 
connected to $i-1$, so that $i-1$ and $i+4$ are black and $i+3$ is white. Since $j$ is connected to $i-1$, we have that $2 \leq d(i, i+4)=d(j, i+4) \leq 2$. Hence, vertex $i-1$ is connected to $i+3$ and to $i+4$. Depending on which edge belongs to $G$, either $(i-1, i+1)$ or $(i, i+2)$, we have Cases (a)-(b) of Fig. 11. Conversely, if Cases (a) or (b) hold, then for any black vertex $i^{\prime} \in\left[i_{1}, i_{2}\right]$, the distances from $i^{\prime}$ to $i$ and $j$ are equal because the shortest path from $i^{\prime}$ to $i$ or $j$ goes through either $i-1$ or $i+4$, and in these cases, the distances from $i$ and $j$ to $i-1$ (resp. to $i+4)$ are the same. Thus, $r\left(i \mid S^{\prime}\right)=r\left(j \mid S^{\prime}\right)$. For the other case, that is, when $j=i-2$ and $j$ is connected to $i+1$, we have by symmetry that $r\left(i \mid S^{\prime}\right)=r\left(j \mid S^{\prime}\right)$ if and only if Cases (c)-(d) of Fig. 11 hold. Therefore, we have proved (1).

We now prove item (2). By Lemma 1, it is clear that two white vertices $i$ and $i+1$ belonging to the same white run are resolved by either $i-1$ or $i+2$. Suppose now that $i$ and $j$ belong to different white runs of size 2 and $r\left(i \mid S^{\prime}\right)=r\left(j \mid S^{\prime}\right)$. In such a case, by Lemma 1 , if $i-1$ is a black vertex, then $j-1$ is a black vertex, and there is an edge connecting $i$ and $j-1$ and another edge connecting $j$ and $i-1$. Also notice that, because of the assumption made in the hypothesis, $i+2$ and $j+2$ are black vertices. Since $2 \leq d(i, j+2)=d(j, j+2) \leq 2$, the only possibility for these two distances to be equal is that vertex $i-1$ is connected to both $j+1$ and $j+2$. Analogously, taking into account that $2 \leq d(j, i+2)=d(i, i+2) \leq 2$, we derive that vertex $j-1$ must be connected to $i+1$ and $i+2$. Depending on which edge belongs to $G$, either $(i, j)$ or $(i-1, j-1)$, we have Cases (e)-(f) in Fig. 11. Conversely, if Cases (e)-(f) hold, then one can easily check that $r\left(i \mid S^{\prime}\right)=r\left(j \mid S^{\prime}\right)$, as there are always shortest paths from $i$ and $j$ to any other black vertex passing through either $i-1$ or $j-1$. Cases (g)-(h) of Fig. 11 appear by symmetry when $i+1$ is black instead of $i-1$. Therefore, (2) follows. Finally, item (3) is a direct consequence of items (1) and (2).

Before proving the main result of this section, we give some additional definitions. The vertex of degree two of Case (c) of Fig. 11 will be called a special vertex. Let $G=(V, E)$ be a graph. Given two subsets $S \subset V$ and $W \subset V$ we say that $S$ discriminates $W$ if every pair of distinct vertices with at least one of them belonging to $W$ is resolved by some vertex in $S$. Note that this condition is stronger than saying that $S$ resolves $W$, used when every pair of vertices in $W$ are resolved by a vertex in $S$ (see [20,21]). Observe that, by definition, if $S_{1}$ discriminates $W_{1}$ and $S_{2}$ discriminates $W_{2}$, then $S_{1} \cup S_{2}$ discriminates $W_{1} \cup W_{2}$. We next prove another technical lemma and the main result of this section, Theorem 4 .

Lemma 3 If $G=(V, E)$ is a MOP graph of ordern, then there is a set $S_{0} \subset V=[1, n]$ of black vertices such that $\left|S_{0}\right|=\left\lceil\frac{2 n}{5}\right\rceil,\{2\}$ is a white run of size 1 , the interval $[4, n]$ is $(1,2)$-alternating and $S_{0}$ discriminates the white run $\{2\}$.

Proof Suppose that $n=5 k+t, t \in\{0,1,2,3,4\}$, for some $k \geq 1$. If $t \in\{0,1,2,3\}$, we begin defining $S_{0}$ as the set of size $\left\lceil\frac{2 n}{5}\right\rceil$ that consists of all the vertices of $[1, n]$ of the form $5 j+1$ and $5 j+3$. Vertices in $S_{0}$ are colored black and the rest, white. We can assume that $S_{0}$ discriminates $\{2\}$. Indeed, if $\{2\}$ is discriminated by $S_{0}$ with the considered numbering of the vertices, we are done. Otherwise, by Lemmas 1 and 2 , the set $\{2\}$ is the white run of size 1 of some of the subgraphs (a)-(d) of Fig. 11. 
In this case, we renumber all the vertices by increasing the corresponding labels by one unit and update the set $S_{0}$ according to the new numbering (that is $S_{0}$ contains the vertices with new labels of the form $5 j+1$ and $5 j+3$ ). Then, the new vertex with label 2 forms a white run of size 1 not belonging to any of the subgraphs (a)-(d) of Fig. 11. Hence, $S_{0}$ discriminates $\{2\}$. If $t=4$, we define $S_{0}$ as the set formed by vertex 1 and all the vertices of $[1, n]$ of the form $5 j+3$ and $5 j+5$, with $j \geq 0$. Then, $\{n\}$, $\{2\}$ and $\{4\}$ are three consecutive white runs of size 1 separated by black vertices. By Lemma $1, S_{0}$ discriminates $\{2\}$, and the interval $[4, n]$ is $(1,2)$-alternating.

Theorem 4 If $G=(V, E)$ is a MOP graph, then there exists a resolving set $S \subset V$ such that $|S|=\left\lceil\frac{2 n}{5}\right\rceil$. Moreover, $S$ can be computed in linear time.

Proof The general procedure to obtain a resolving set for $G$ is the following. We begin with the set $S:=S_{0}$ defined in the proof of Lemma 3 that discriminates the white run $\{2\}$ of size 1 . If $S_{0}$ is a resolving set for $G$, we are done. Otherwise, we explore clockwise the white runs of $S$. Suppose that, after exploring the first $h$ runs, $S$ is a set consisting of $\left\lceil\frac{2 n}{5}\right\rceil$ vertices which is a candidate to be a resolving set for $G$. If the next white run is not discriminated by $S$ because it belongs to some of the subgraphs shown in Fig. 11, then we define a new set $S^{\prime}$ by removing some vertices in $S$ and including new ones, such that $\left|S^{\prime}\right|=|S|$ and all explored white runs are discriminated by $S^{\prime}$. Then, we update the set $S, S:=S^{\prime}$, and continue the exploration.

More precisely, suppose that, in a generic step of the exploration, the vertices in the interval $I=[1, i-1]$ have already been explored. Then, we denote by $S$ the set of $\left\lceil\frac{2 n}{5}\right\rceil$ black vertices of $G$ and by $W$ the set of white vertices of $I$, that is, $W=I \backslash S$.

We then prove that $I$ and $S$ satisfy the following invariant.

Invariant 1. If $I=[1, i-1], S \subseteq V$ and $W=I \backslash S$, then:

Property $P 1 \quad|S|=\left\lceil\frac{2 n}{5}\right\rceil$ and $S$ discriminates $W$.

Property $P 2$ Vertex $i$ is white, vertices 1 and $i-1$ are black (that is, $\{1, i-1\} \subseteq S$ and $i \notin S)$, and $[i, n]$ is a $(1,2)$-alternating interval.

Property P3 For every white vertex $w \in W \backslash\{i-2\}$ and every white special vertex $l \in V \backslash I$, there exists a black vertex $v \in S \cap(I \backslash\{i-1\})$ such that $v$ resolves $w$ and $l$.

Obviously, by Property P1, $S$ will be a resolving set of size $\left\lceil\frac{2 n}{5}\right\rceil$ for $G$ after exploring all white runs. Properties P2 and P3 are technical facts that will be needed to proceed with the proof.

We begin verifying that Invariant 1 holds for $S=S_{0}$ and $I=[1,3]$. By Lemma 3, $S_{0}$ discriminates $\{2\}$, and the interval $[4, n]$ is $(1,2)$-alternating. Thus, Property P2 obviously holds and Property P3 is true because in this case the set $W \backslash\{2\}=\emptyset$.

Assuming that Invariant 1 is true for given sets $I=[1, i-1]$ and $S$, we next show that it holds for new sets $I^{\prime}$ and $S^{\prime}$ defined after exploring clockwise the next white run $r$ not belonging to $I$. We will distinguish whether $r$ is already discriminated by $S$ or not.

Suppose first that $r$ is discriminated by $S$. If $r$ only consists of the white vertex $i$, then one can easily check that Invariant 1 holds for $S^{\prime}=S$ and $I^{\prime}=I \cup[i, i+1]$. Indeed, as $S$ discriminates $W$ and $i$, then $S$ obviously discriminates $W^{\prime}=W \cup\{i\}$. Property P3 follows from the fact that a vertex in $W \backslash\{i-2\}$ satisfies Property P3, and 


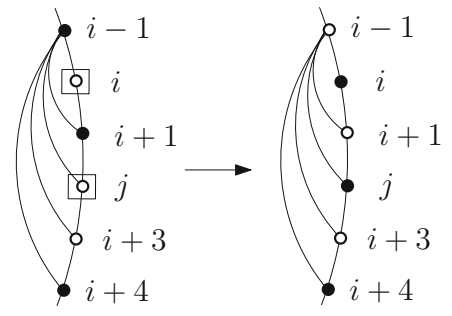

(a)

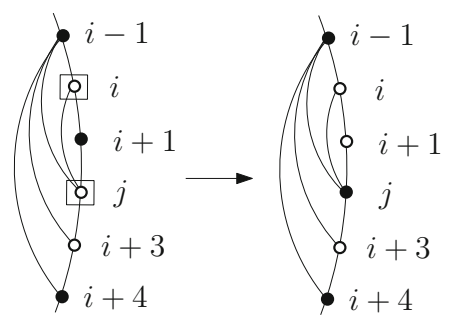

(b)

Fig. 12 Squared vertices have the same coordinates. Case (a): interchanging the colors of $i$ and $i-1$, and $j=i+2$ and $i+1$. Case (b): interchanging the colors of $i+1$ and $j=i+2$

a special vertex $l$ cannot be connected to $i-1$, so $i-1$ resolves $i-2$ and $l$. Hence, $I^{\prime}$ satisfies Property P3. If $r$ consists of two white vertices, $i, i+1$, consider $S^{\prime}=S$ and $I^{\prime}=I \cup[i, i+2]$. Sets $S^{\prime}$ and $I^{\prime}$ satisfy Property P1 of Invariant 1 , because $S$ discriminates $W^{\prime}=W \cup\{i, i+1\}$. To see that Property P3 is true, notice that a special vertex $l \in V \backslash I^{\prime}$ is not connected to $i-1$. Hence, $i-1$ resolves $l$ and any of $i-2$ and $i$.

Suppose now that $r$ is not discriminated by $S$. As $S$ discriminates $W$, the white vertices not resolved by $S$ must belong to the interval $[i, n]$. Lemma 2 can be applied to the interval $[i-1,1]$, if $n$ is white, or to the interval $[i-1, n]$, if $n$ is black, since the interval $[i, n]$ is $(1,2)$-alternating by Property 2 . Hence, $r$ belongs to one of the subgraphs of Cases (a)-(h). Note that if $r$ has size 1, then $r$ consists of vertex $i$, and if $r$ has size 2 , then $r$ consists of vertices $i$ and $i+1$.

In each one of these 8 cases, the general framework to construct new sets $S^{\prime}$ and $I^{\prime}$ satisfying Invariant 1 is the following. The set $I^{\prime}$ is obtained by adding an interval $\left[i, i^{\prime}\right]$ to $I$, where $i^{\prime}$ is a black vertex and the vertices of the run $r$ are in $\left[i, i^{\prime}\right]$. Then, we interchange the colors of some vertices from $\left[i-1, i^{\prime}\right]$, so that the updated set $S^{\prime}$ of black vertices satisfies $\left|S^{\prime}\right|=|S|=\left\lceil\frac{2 n}{5}\right\rceil$, and Invariant 1 holds for the new sets $I^{\prime}=I \cup\left[i, i^{\prime}\right]$ and $S^{\prime}$. To complete the validity of Property P1, it is needed to show that $S^{\prime}$ discriminates $W^{\prime}$ after interchanging some colors in $I^{\prime}$. This will be proved in two steps. First, we give a subset of $S^{\prime}$ that discriminates the set of new white vertices, $X=W^{\prime} \backslash W$. Secondly, we show that every pair of white vertices $x$ and $y$, with $x \in W$ and $y \in V \backslash X$, that was resolved by a vertex from $S \backslash S^{\prime}$, is now resolved by a vertex from $S^{\prime} \backslash S$. Property P2 follows, because we have not changed the colors of the vertices from $V \backslash I^{\prime}$. Finally, to prove that the sets $S^{\prime}$ and $I^{\prime}$ satisfy Property P3, it is enough to show that it holds for the white vertices in $\left(X \backslash\left\{i^{\prime}-1\right\}\right) \cup\{i-2\}$. We next analyze the different cases.

Case (a) The two vertices not resolved by $\mathrm{S}$ are $\mathrm{i}$ and $\mathrm{j}$ as shown in Fig. 12a. In this case, we interchange the colors of vertices $i$ and $i-1$ and the colors of the vertices $j(=i+2)$ and $i+1$. We claim that Invariant 1 holds for the new sets $I^{\prime}=I \cup[i, i+4]$ and $S^{\prime}=(S \backslash\{i-1, i+1\}) \cup\{i, i+2\}$.

Let us see that $S^{\prime}$ discriminates $W^{\prime}=W \cup\{i-1, i+1, i+3\}$. On the one hand, the set $S^{*}=\{i, i+2, i+4\}$ discriminates $\{i-1, i+1, i+3\}$, because $r\left(i-1 \mid S^{*}\right)=(1,1,1)$, $r\left(i+1 \mid S^{*}\right)=(1,1,2)$ and $r\left(i+3 \mid S^{*}\right)=(2,1,1)$, respectively, and the only vertices 


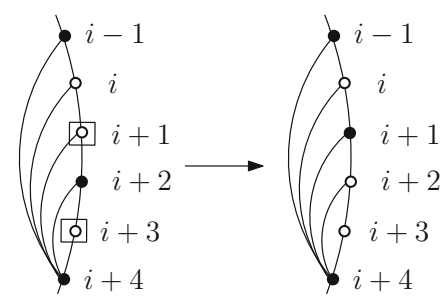

(c)

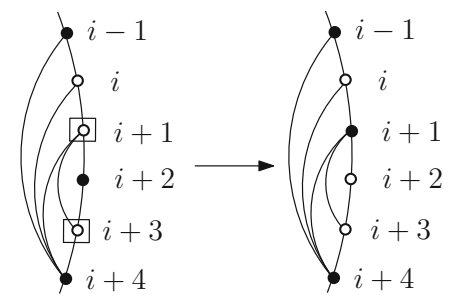

(d)

Fig. 13 Squared vertices have the same coordinates. Case (c): Interchanging the colors of $i+1$ and $i+2$, when $\{i+3\}$ is discriminated by $(S \backslash\{i+2\}) \cup\{i+1\}$. Case (d): Interchanging the colors of $i+1$ and $i+2$

adjacent to $i+2$ are precisely $i-1, i+1$ and $i+3$. On the other hand, the shortest path from $i^{\prime} \in[i+5, i-2]$ to $i, i+1$ or $i+2$ necessarily goes through $i-1$, so $d\left(i^{\prime}, i\right)=d\left(i^{\prime}, i-1\right)+1$ and $d\left(i^{\prime}, i+2\right)=d\left(i^{\prime}, i+1\right)$. This implies that if $i-1$ (resp. $i+1$ ) resolves two vertices in $[i+5, i-2]$ then $i$ (resp. $i+2$ ) also resolves them. In particular, $S^{\prime}$ discriminates $W^{\prime}$.

Let us see that Property P3 also holds. Take a special white vertex $l$ in $V \backslash I^{\prime}$. Property $\mathrm{P} 3$ clearly holds for the vertices of $W \backslash\{i-2\}$. Moreover, since $d(l, i+2) \geq 3$ and the distance from $i+2$ to any of $\{i-2, i-1, i+1\}$ is at most two, then we have that $i+2$ resolves $l$ and any white vertex of $\{i-2, i-1, i+1\}$. Therefore, Property P3 is satisfied, and Invariant 1 holds as claimed.

Case (b) The two vertices not resolved by $\mathrm{S}$ are $\mathrm{i}$ and $\mathrm{j}$ as shown in Fig. 12b. In this case, we only need to interchange the colors of vertices $j(=i+2)$ and $i+1$. We claim that Invariant 1 holds for the new sets $I^{\prime}=[1, i+4]$ and $S^{\prime}=(S \backslash\{i+1\}) \cup\{i+2\}$. Notice that $W^{\prime}=W \cup\{i, i+1, i+3\}$.

The set $S^{*}=\{i+2, i+4\}$ discriminates $\{i, i+1, i+3\}$ because $r\left(i \mid S^{*}\right)=(1,2)$, $r\left(i+1 \mid S^{*}\right)=(1,3)$ and $r\left(i+3 \mid S^{*}\right)=(1,1)$, and the only white vertices adjacent to $i+2$ are precisely $i, i+1$ and $i+3$ (see Fig. 12b). Moreover, observe that for a vertex $i^{\prime} \in[i+5, i-2]$, we have $d\left(i^{\prime}, i+2\right)=d\left(i^{\prime}, i+1\right)-1$, so if $i+1$ resolves two vertices in $[i+5, i-2]$, then $i+2$ resolves them as well. As a consequence, $S^{\prime}$ discriminates $W$.

Finally, to prove Property $\mathrm{P} 3$, note that the distance from $i+2$ to a special vertex $l \in V \backslash I^{\prime}$ is at least 3. Thus, $i+2$ resolves the pairs formed by $l$ and a vertex from $\{i-2, i, i+1\}$.

Case (c) In this case, the vertices not resolved by $S$ are $i+1$ and $i+3$ (see Fig. 13c). We begin by interchanging the colors of the vertices $i+1$ and $i+2$, and distinguish two cases depending on whether $(S \backslash\{i+2\}) \cup\{i+1\}$ discriminates $\{i+3\}$ or not.

Suppose first that $\{i+3\}$ is discriminated by $(S \backslash\{i+2\}) \cup\{i+1\}$. Then, Invariant 1 holds for the sets $S^{\prime}=(S \backslash\{i+2\}) \cup\{i+1\}$ and $I^{\prime}=I \cup[i, i+4]$. Note that $W^{\prime}=W \cup\{i, i+2, i+3\}$. Indeed, observe that $S^{*}=\{i-1, i+1\}$ discriminates $\{i, i+2\}$, because $r\left(i \mid S^{*}\right)=(1,1), r\left(i+2 \mid S^{*}\right)=(2,1)$, and the only white vertices at distance 1 from $i+1$ are $i$ and $i+2$. Besides, $d\left(i^{\prime}, i+2\right)=d\left(i^{\prime}, i+1\right)$ for every vertex $i^{\prime} \in[i+5, i-2]$, implying that every pair of vertices belonging to $[i+5, i-2]$ that were resolved by $i+2$ are now resolved by $i+1$. In particular, $S^{\prime}$ discriminates 
Fig. 14 Case (c): Squared vertices have the same coordinates. When $\{i+3\}$ is not discriminated by

$(S \backslash\{i+2\}) \cup\{i+1\}$, the colors of $i+3$ and $i+4$ are interchanged
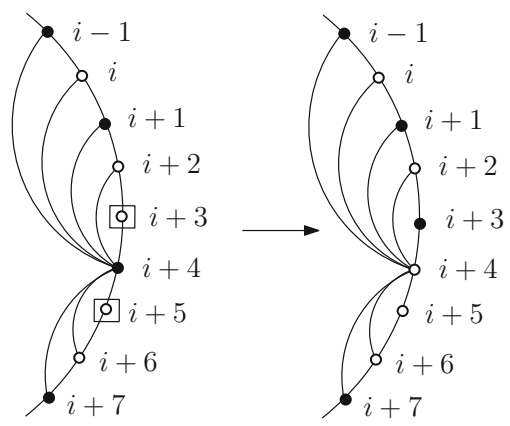

$W$. Therefore, $S^{\prime}$ discriminates $W^{\prime}$, and Property P1 holds. Since a special vertex $l$ in $V \backslash I^{\prime}$ is not connected to either $i-1$ or $i+1$, then $l$ together with a vertex from $\{i-2, i, i+2\}$ are resolved by either $i-1$ or $i+1$. Then, Property P3 also holds.

Suppose now that $(S \backslash\{i+2\}) \cup\{i+1\}$ does not discriminate $\{i+3\}$. Let us see which vertex $j$ has the same coordinates as $i+3$ in relation to this set. Notice that by Property 3, since $i+3$ is a special vertex, for any vertex $j \in W \backslash\{i-2\}$ there is a vertex in $I \cap S$ resolving $i+3$ and $j$. Besides, $i-1$ resolves the pair $i+3$ and $i-2$, and $i+1$ resolves $i+3$ and any of $i$ and $i+2$. Hence, $j \notin[1, i+2]$. By Property $\mathrm{P} 2$, a vertex $j \in[i+5, n]$ is adjacent to a black vertex $j^{\prime}$, but $j^{\prime}$ is not adjacent to $i+3$ unless $j^{\prime}=i+4$ and $j=i+5$. Then, $i+6$ is white and $i+7$ is black (see Fig. 14). Since $2 \leq d(i+3, i+7)$ and $d(i+5, i+7) \leq 2$, we have that both distances are equal only when the edges $(i+4, i+7)$ and $(i+4, i+6)$ belong to $\mathrm{G}$.

If this situation happens, then $i+3$ and $i+5$ have the same coordinates in relation to $(S \backslash\{i+2\}) \cup\{i+1\}$. We remark that Property P3 is important at this time to ensure that $i+5$ is the only vertex with the same coordinates as $i+3$. Otherwise, if Property P3 does not hold, then a vertex $x$ in $W$ could have the same coordinates as $i+3$, because $i+2$ could be the only vertex in $S$ to resolve $i+3$ and $x$.

We interchange the colors of vertices $i+3$ and $i+4$, as shown in Fig. 14, and set $I^{\prime}=[1, i+7]$ and $S^{\prime}=(S \backslash\{i+2, i+4\}) \cup\{i+1, i+3\}$. Thus, $W^{\prime}=$ $W \cup\{i, i+2, i+4, i+5, i+6\}$. The argument to prove that Invariant 1 holds for these new sets is similar to the previous ones, but a bit more elaborated.

Let us show that $S^{\prime}$ discriminates $W^{\prime}$. On the one hand, if $S^{*}=\{i-1, i+1, i+3\} \subseteq$ $S^{\prime}$, then $r\left(i \mid S^{*}\right)=(1,1,2), r\left(i+2 \mid S^{*}\right)=(2,1,1)$ and $r\left(i+4 \mid S^{*}\right)=(1,1,1)$. In addition, the only vertices at distance 1 from $i+1$ are $i, i+2$ and $i+4$. Hence, $S^{\prime}$ discriminates $\{i, i+2, i+4\}$. On the other hand, for every vertex $i^{\prime} \in[i+5, i-2]$, we have $d\left(i^{\prime}, i+2\right)=d\left(i^{\prime}, i+1\right)$ and $d\left(i^{\prime}, i+3\right)=d\left(i^{\prime}, i+4\right)+1$. This implies that any pair of vertices from $[i+5, i-2]$ resolved by $i+2$ or $i+4$ is also resolved by $i+1$ or $i+3$. Therefore, since $W \subseteq[i+5, i-2]$ and $S$ discriminates $W$, we derive that $S^{\prime}$ discriminates $W$. It only remains to prove that $S^{\prime}$ discriminates $\{i+5, i+6\}$. Notice that $i+7$ resolves the pair $i+5$ and $i+6$. Moreover, by Property $\mathrm{P} 2$, a white vertex $j$ in $V \backslash I^{\prime}$ is adjacent to a black vertex $j^{\prime}$. Since $i+5$ and $i+6$ cannot be connected to $j^{\prime}$, then $j^{\prime}$ resolves $j$ and any vertex of $i+5$ and $i+6$. Note that if $j=i+8$, then $i+9$ is such a vertex $j^{\prime}$. Finally, as $S^{\prime}$ discriminates $W$ and $\{i, i+2, i+4\}$, a 
vertex from $\{i+5, i+6\}$ and a vertex from [1,i+4] are resolved by some vertex of $S^{\prime}$. Hence, Property P1 is satisfied.

To show that Property P3 holds, we only need to prove this property for the vertices $i-2, i, i+2, i+4$ and $i+5$. For a special vertex $l$ in $V \backslash I^{\prime}$, its distance to $i+3$ is at least 3 . Since the distance from $i+3$ to $i, i+2, i+4$, or $i+5$ is at most 2 , vertex $i+3$ resolves $l$ and any of these four vertices. The pair $l$ and $i-2$ is resolved by $i-1$, because $l$ is not adjacent to $i-1$.

Case (d) In this case, the vertices not resolved by $S$ are $i+1$ and $i+3$ in the subgraph shown in Fig. 13d. This case is symmetric to Case (b). Following the same kind of arguments used in that case, one can easily prove that Invariant 1 holds for the sets $I^{\prime}=[1, i+4]$ and $S^{\prime}=(S \backslash\{i+2\}) \cup\{i+1\}$, defined after interchanging the colors of vertices $i+1$ and $i+2$ (see Fig. 13d).

Case (e) In this case, the vertices not resolved by $S$ are $i$ and $j$ in the subgraph shown in Fig. 15e. We interchange the colors of vertices $i-1$ and $i$ and we define $I^{\prime}=I \cup[i, i+2]$ and $S^{\prime}=(S \backslash\{i-1\}) \cup\{i\}$. Thus, $W^{\prime}=W \cup\{i-1, i+1\}$ (see Fig. 15e).

Let us see first that $S^{\prime}$ discriminates $W^{\prime}$. On the one hand, the set $S^{*}=\{i, i+2\}$ discriminates $\{i-1, i+1\}$. Indeed, $r\left(i-1 \mid S^{*}\right)=(1,3), r\left(i+1 \mid S^{*}\right)=(1,1)$, and the only white vertices belonging to $V$ at distance 1 from $i$ are $i-1, i+1$ and $j$, but $r\left(j \mid S^{*}\right)=(1,2)$. On the other hand, $d\left(i^{\prime}, i\right)=d\left(i^{\prime}, i-1\right)+1$ for every vertex $i^{\prime} \in[j+1, i-2]$. Hence, since $W \subseteq[j+3, i-2]$, every pair of vertices with at least one of them belonging to $W$ and the other to $[j+3, i-2]$ that was resolved by $i-1 \in S$ is now resolved by $i \in S^{\prime}$. It only remains to prove that every pair formed by a vertex $i^{\prime}$ from $W$ and a white vertex $j^{\prime} \in[i+3, j]$ is resolved by some vertex of $S^{\prime}$. This is true because, by Property $\mathrm{P} 2, j^{\prime}$ is adjacent to a black vertex in $S^{\prime} \cap[i+3, j]$ that is not adjacent to $i^{\prime}$. Hence, Property P1 holds. To prove property P3, it suffices to check that it holds for the vertices $i-1$ and $i-2$. If $l \in V \backslash I^{\prime}$ is a special vertex different from $j-2$, then $d(l, i) \geq 3$. Hence, the pairs formed by $l$ and a vertex from $\{i-1, i-2\}$ are resolved by $i$, whenever $l \neq j-2$. Suppose that $l=j-2$ is a special vertex. If we take a black vertex $j^{\prime \prime} \neq i-1$ in $I$, then $d\left(j-2, j^{\prime \prime}\right)=3+d\left(i-1, j^{\prime \prime}\right)$ and $d\left(i-2, j^{\prime \prime}\right) \leq d(i-2, i-1)+d\left(i-1, j^{\prime \prime}\right)=1+d\left(i-1, j^{\prime \prime}\right)$. Hence, $j^{\prime \prime} \in I^{\prime}$ resolves $j-2$ and any of $i-1$ and $i-2$.

Case (f) In this case, the vertices not resolved by $S$ are $i$ and $j$ in the subgraph shown in Fig. 15f. This case is very similar to the previous one. By interchanging the colors of vertices $i-1$ and $i$ (see Fig. 15f), the proof that sets $I^{\prime}=[1, i+2]$, and $S^{\prime}=(S \backslash\{i-1\}) \cup\{i\}$ satisfy Invariant 1 is essentially the same as the proof done in Case (e), with small differences due to the fact that the edge $(i-1, j-1)$ now belongs to $G$ instead of edge $(i, j)$.

Case (g) In this case, the vertices not resolved by $S$ are $i+1$ and $j+1$ in the subgraphs shown in Fig. 16. We begin by interchanging the colors of vertices $i+1$ and $i+2$. We distinguish two cases depending on whether $(S \backslash\{i+2\}) \cup\{i+1\}$ discriminates $\{i+3\}$ or not.

Suppose first that $(S \backslash\{i+2\}) \cup\{i+1\}$ discriminates $\{i+3\}$ (see Fig. 16, top). We claim that $S^{\prime}=(S \backslash\{i+2\}) \cup\{i+1\}$ and $I^{\prime}=I \cup[i, i+4]$ satisfy Invariant 1 . Note that $W^{\prime}=W \cup\{i, i+2, i+3\}$. 


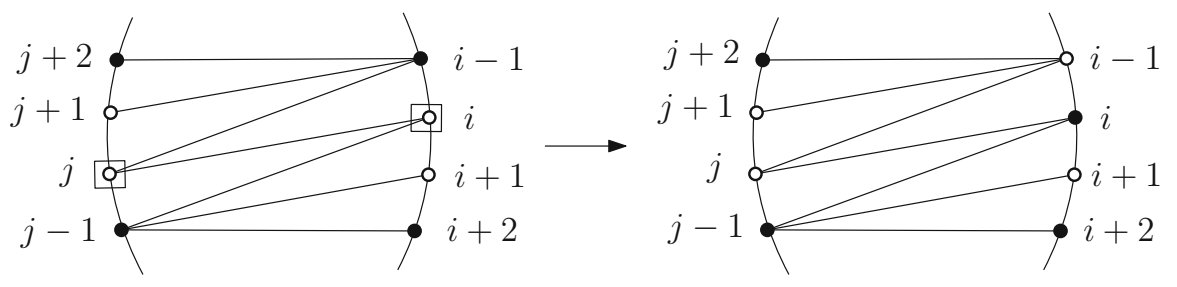

(e)
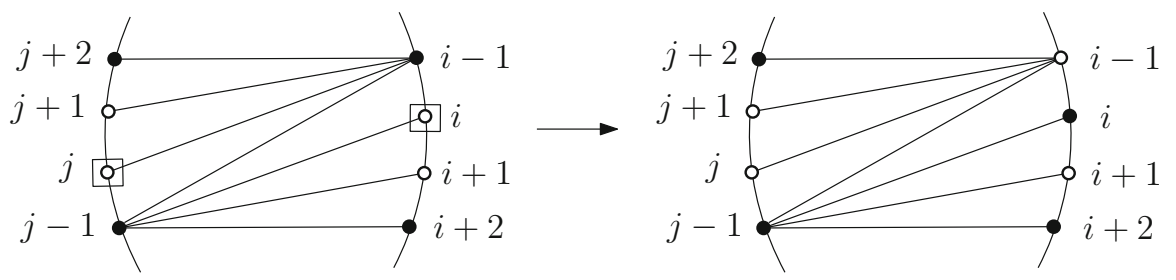

(f)

Fig. 15 Cases (e) and (f): squared vertices have the same coordinates. Interchanging the colors of $i-1$ and $i$

On the one hand, the set $S^{*}=\{i-1, i+1\}$ discriminates $\{i, i+2, j+1\}$. Indeed, $r\left(i \mid S^{*}\right)=(1,1), r\left(i+2 \mid S^{*}\right)=(3,1), r\left(j+1 \mid S^{*}\right)=(2,1)$ and the only white vertices adjacent to $i+1$ are $i, i+2$ and $j+1$. We include here vertex $j+1$ to ensure that $j+1$ and a vertex in $W$ are resolved. On the other hand, a white vertex $i^{\prime} \in[i+4, j]$ is adjacent by Property $\mathrm{P} 2$ to a black vertex $j^{\prime}$ in this interval. Thus, $j^{\prime}$ resolves any pair formed by $i^{\prime}$ together with every white vertex of $W \subseteq[j+3, i-2]$ because the vertices of this last interval are not adjacent to $j^{\prime}$. In addition, since $d\left(i^{\prime}, i+1\right)=d\left(i^{\prime}, i+2\right)-1$ for every vertex $i^{\prime} \in[j+3, i-1]$ and $W \subseteq[j+3, i-1]$, every pair of vertices in this interval, with one of them in $W$, that was resolved by $i+2$ is now resolved by $i+1$. Hence, $S^{\prime}$ discriminates $W$. Besides, since a special white vertex $l$ is not connected to either $i+1$ or $i-1$, and vertices $i-2, i$ and $i+2$ are adjacent to at least one of them, Property P3 holds.

Suppose now that $(S \backslash\{i+2\}) \cup\{i+1\}$ does not discriminate $\{i+3\}$. Let us see which white vertex $j^{\prime}$ has the same coordinates as $i+3$ with respect to this set. A vertex in the interval $[j, i+1]$ is not adjacent $i+4$; thus, $j^{\prime} \in[i+2, j-2]$. Moreover, $j^{\prime} \neq i+2$, because $i+3$ is not adjacent to $i+1$, and consequently, $j^{\prime} \in[i+5, j-2]$. Observe now that if $d(i+3, i+1)=d\left(j^{\prime}, i+1\right)=2$ then $d(i+3, i+2)=d\left(j^{\prime}, i+2\right)=1$. Taking vertex $i+2$ as a black vertex, we can apply Lemma 2 to the $(1,2)$-alternating interval $[i+2, n]$ (or $[i+2,1])$, giving rise to the only two possibilities shown in Fig. 16, middle and bottom, for a vertex $j^{\prime}=i+5$ to have the same coordinates as $i+3$.

Consider the case shown in Fig. 16, middle: vertex $i+2$ connected to vertices $i+3, i+4, i+5, i+6$ and $i+7$. In addition to the changes of color of $i+1$ and $i+2$, we interchange the colors of vertices $i+4$ and $i+5$. We claim that the sets $S^{\prime}=(S \backslash\{i+2, i+4\}) \cup\{i+1, i+5\}$ and $I^{\prime}=I \cup[i, i+7]$ satisfy Invariant 1 . Note that $W^{\prime}=W \cup\{i, i+2, i+3, i+4, i+6\}$. The set $S^{*}=\{i-1, i+1, i+5, i+7\}$ 

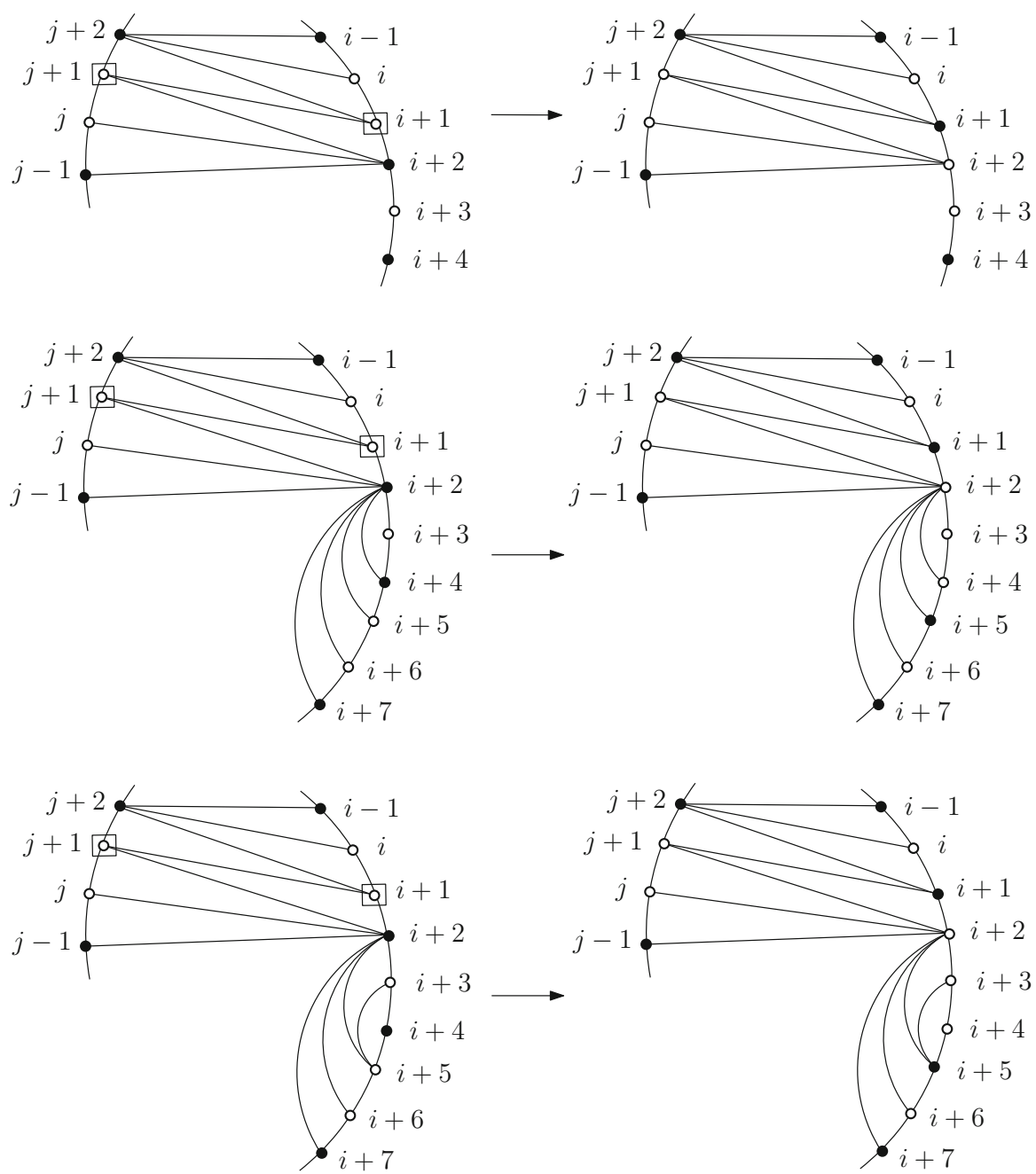

Fig. 16 Case (g): Squared vertices have the same coordinates. Top: Interchanging the colors of $i+1$ and $i+2$. Middle and bottom: Interchanging the colors of $i+1$ and $i+2$ and the colors of $i+4$ and $i+5$

discriminates $\{i, i+2, i+4, i+6, j+1\}$, since $\{i, i+2, i+4, i+6, j+1\}$ are the only white vertices adjacent to $i+1$ or $i+5$ and $r\left(i \mid S^{*}\right)=(1,1,3,3)$, $r\left(i+2 \mid S^{*}\right)=(3,1,1,1), r\left(i+4 \mid S^{*}\right)=(4,2,1,2), r\left(i+6 \mid S^{*}\right)=(4,2,1,1)$, and $r\left(j+1 \mid S^{*}\right)=(2,1,2,2)$. On the other hand, $i+3$ has no black neighbor. Hence, any other white vertex $i^{\prime} \in V \backslash W^{\prime}$ has at least a black neighbor that resolves $i+3$ and $i^{\prime}$. If $i^{\prime} \in W \subseteq[j+3, i-2]$, then $i+5$ resolves $i^{\prime}$ and $i+3$, because $d\left(i^{\prime}, i+5\right) \geq 4$, but $d(i+3, i+5)=2$. Thus, $S^{\prime}$ discriminates $\{i+3\}$. Finally, as $S$ discriminates $W$ and $W \subseteq[j+3, i-2]$, then $S^{\prime}$ also discriminates $W$ taking into account that (1) $j+1$ is already resolved from a vertex in $W,(2)$ for every vertex $i^{\prime} \in[j+3, i-2]$, we have $d\left(i^{\prime}, i+5\right)=d\left(i^{\prime}, i+4\right)$ and $d\left(i^{\prime}, i+1\right)=d\left(i^{\prime}, i+2\right)-1$ and that (3) 
a black vertex adjacent to a white vertex of $V \backslash I^{\prime}$ in the interval $[i+8, j]$ cannot be connected to a vertex in $W$. Therefore, $S^{\prime}$ discriminates $W^{\prime}$ and Property P1 holds.

To show that Property P3 holds we only need to resolve the pairs formed by a special vertex $l \in V \backslash I^{\prime}$ and one of the vertices from $\{i-2, i, i+2, i+3, i+4\}$ using a vertex from $S^{\prime} \cap[1, i+5]$. Vertex $i-1$ resolves $l$ and any of $\{i-2, i\}$ because $l$ is not adjacent to $i-1$, and $i+5$ resolves $l$ and any of $\{i+2, i+3, i+4\}$ because $d(l, i+5) \geq 3$. Therefore, Property P3 also holds, and Invariant 1 is satisfied, as claimed.

For the last case, the one shown in Fig. 16, bottom, the analysis is very similar to the previous one. Following the same steps as described in the two previous paragraphs, one can prove that $S^{\prime}=(S \backslash\{i+2, i+4\}) \cup\{i+1, i+5\}$ and $I^{\prime}=I \cup[i, i+7]$ satisfy Invariant 1 . The set $W^{\prime}$ is $W \cup\{i, i+2, i+3, i+4, i+6\}$. In this case, it can be shown that the set $S^{*}=\{i-1, i+1, i+5, i+7\}$ discriminates $\{i, i+2, i+3, i+4, i+6, j+1\}$. Moreover, for every white vertex $i^{\prime} \in[j+3, i-2]$, we have $d\left(i^{\prime}, i+1\right)=d\left(i^{\prime}, i+2\right)-1$ and $d\left(i^{\prime}, i+5\right)=d\left(i^{\prime}, i+4\right)-1$. Thus, every pair of white vertices from $[j+3, i-2]$ that was resolved by $i+2$ or $i+4$ is resolved now by $i+1$ or $i+5$. For every white vertex $i^{\prime} \in[i+8, j]$, the black vertex adjacent to $i^{\prime}$ is not adjacent to a vertex in $W \subseteq[j+3, i-2]$. Hence, $S^{\prime}$ discriminates $W$.

Finally, to show that Property P3 holds we only need to resolve the pairs formed by a special vertex $l \in V \backslash I^{\prime}$ and one of the vertices from $\{i-2, i, i+2, i+3, i+4\}$ using a vertex from $S^{\prime} \cap[1, i+5]$. All the vertices in $\{i-2, i, i+2, i+3, i+4\}$ are adjacent to either $i-1$ or $i+5$, but a special vertex $l \in V \backslash I^{\prime}$ is not adjacent to either $i-1$ or $i+5$, so $i-1$ or $i+5$ resolves $l$ and any of these five vertices. From this, Invariant 1 holds as claimed.

Case (h) In this case, the vertices not resolved by $S$ are $i+1$ and $j+1$ in the subgraphs shown in Fig. 17. The analysis of Case (h) follows the same steps as Case (g), although there are small changes due to the fact that now the edge $(i+2, j+2)$ belongs to $G$ instead of the edge $(i+1, j+1)$.

If $(S \backslash\{i+2\}) \cup\{i+1\}$ discriminates $\{i+3\}$, it can be checked that $S^{\prime}=(S \backslash\{i+2\}) \cup$ $\{i+1\}$ and $I^{\prime}=[1, i+4]$ satisfy Invariant 1 (see Fig. 17, top). If $(S \backslash\{i+2\}) \cup\{i+1\}$ does not discriminate $\{i+3\}$, arguing exactly as in Case $(\mathrm{g})$, we have that the vertex with the same coordinates as $i+3$ is $j^{\prime}=i+5$, and one of the cases shown in the middle and bottom of Fig. 17 holds. It can be checked in both cases that the sets $S^{\prime}=(S \backslash\{i+2, i+4\}) \cup\{i+1, i+5\}$ and $I^{\prime}=I \cup[i, i+7]$ satisfy Invariant 1 (see Fig. 17, middle and bottom).

To finish the proof of the theorem, let us see that $S$ can be computed in linear time. Building $S=S_{0}$ obviously requires linear time. Besides, for every run $r$, we have to check whether subgraphs (a)-(h) appear in $G$ and, if it is the case, to update $S$ accordingly. All of this can be done in constant time. Therefore, $S$ can be computed in linear time.

\section{Conclusions and Open Problems}

In this paper, we have studied the metric dimension problem for maximal outerplanar graphs, and we have shown that $2 \leq \beta(G) \leq\left\lceil\frac{2 n}{5}\right\rceil$ for any maximal outerplanar graph 

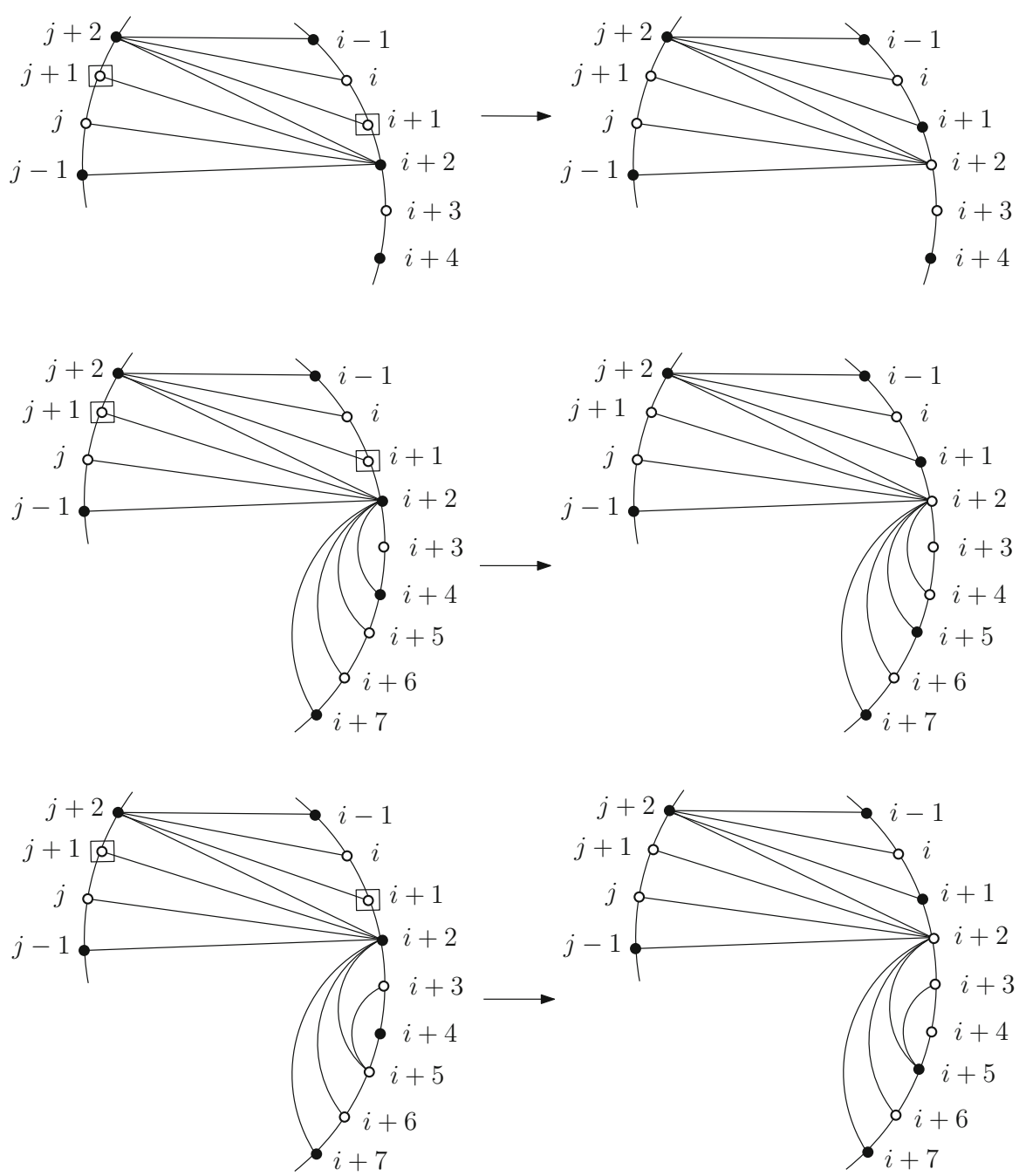

Fig. 17 Case (h): Squared vertices have the same coordinates. Top: Interchanging the colors of $i+1$ and $i+2$. Middle and bottom: Interchanging the colors of $i+1$ and $i+2$ and the colors of $i+4$ and $i+5$

$G$. In relation to the lower bound, we have characterized all maximal outerplanar graphs with metric dimension two, based on embedding such graphs into the strong product of two paths. A first question is whether this technique can be applied to characterize graphs with metric dimension two in other families of graphs, as 2-trees or near-triangulations.

With respect to the upper bound, we have provided a linear algorithm to build a resolving set of size $\left\lceil\frac{2 n}{5}\right\rceil$ for any maximal outerplanar graph. Another question is whether similar techniques as those described in the algorithm can be used to find efficiently resolving sets for other families of graphs, as Hamiltonian outerplanar graphs or 
near-triangulations. For near-triangulations, our conjecture is that there always exists a resolving set of size $\left\lceil\frac{2 n}{5}\right\rceil$ for any near-triangulation.

Finally, let us point out that using similar reasonings, it can be proved that a MOP of order $n \geq 4$ has a locating-dominating set of cardinality $\lfloor n / 2\rfloor$. This fact reinforces the veracity of the conjecture given in [15], stating that every twin-free graph has a locating-dominating set of size at most half the order. Recall that two vertices are twins if they have the same open or closed neighborhood, and a graph is twin-free if it has no twins. Since MOPs of order at least 5 have no twins, the conjecture holds for this class of twin-free graphs.

Proposition 2 If $G$ is a MOP of order at least 4, then $G$ has a locating-dominating set of cardinality $\lfloor n / 2\rfloor$.

Proof There is only one MOP of order 4 and the set consisting of a vertex of degree 2 and a vertex of degree 3 is a locating dominating set. The only MOP of order 5 is a fan, and in this case, the set formed by the two vertices of degree 2 is a locating-dominating set.

For $n \geq 6$, let us number the vertices of the outerface cycle of the MOP with consecutive numbers, from 1 to $n$, so that vertex 1 has degree 2 (note that this is possible since every MOP has at least 2 vertices of degree 2) and consider the set $S$ consisting of even vertices. We claim that $S$ is a locating-dominating set of cardinality $\lfloor n / 2\rfloor$. On the one hand, every vertex not in $S$ has a neighbor in $S$. On the other hand, for every pair of vertices not in $S$, that is, for every pair of odd vertices $i$ and $j$, their sets of neighbors in $S$ are different. Indeed, if either $n$ is odd with $i$ and $j$ different from 1, or $n$ is even, then there are at least three distinct vertices in $S$ that are neighbors of $i$ or $j$. Hence, $i$ and $j$ have different sets of neighbors in $S$ because a MOP does not contain a complete bipartite graph $K_{2,3}$ as subgraph. If $n$ is odd and one of the vertices is 1 , we may assume $i=1$. By construction of $S$, the only neighbor of 1 in $S$ is 2 and any other odd vertex $j$ has at least two neighbors in $S$ (recall that, if $n$ is odd, then $n$ is adjacent to $n-1$ and 2, both in $S$ ). Hence, the set of neighbors of 1 and $j$ in $S$ are different.

Acknowledgements The authors would like to thank the anonymous referees for valuable comments which helped to improve the paper. A. García, M. Mora and J. Tejel are supported by H2020-MSCA-RISE project 734922-CONNECT; M. Claverol, A. García, G. Hernández, C. Hernando, M. Mora and J. Tejel are supported by Project MTM2015-63791-R (MINECO/FEDER); M. Claverol, C. Hernando, M. Mora and J. Tejel are supported by project PID2019-104129GB-I00/AEI/10.13039/501100011033 of the Spanish Ministry of Science and Innovation; M. Claverol is supported by project Gen. Cat. DGR 2017SGR1640; C. Hernando, M. Maureso and M. Mora are supported by project Gen. Cat. DGR 2017SGR1336; and A. García and J. Tejel are supported by project Gobierno de Aragón E41-17R (FEDER).

\section{References}

1. Beaudou, L., Dankelmann, P., Florent, F., Henning, M.A., Mary, A., Parreau, A.: Bounding the order of a graph using its diameter and metric dimension: a study through tree decompositions and VC dimension. SIAM J. Discrete Math. 32(2), 902-918 (2018)

2. Beerliova, Z., Eberhard, F., Erlebach, T., Hall, A., Hoffman, M., Mihalák, M., Ram, L.S.: Network discovery and verification. IEEE J. Sel. Areas Commun. 24, 2168-2181 (2006) 
3. Behtoei, A., Davoodi, A., Jannesari, M., Omoomi, B.: A characterization of some graphs with metric dimension two. Discrete Math. Algorithms Appl. 9, 175027 (2017). (15 pages)

4. Cáceres, J., Hernando, C., Mora, M., Pelayo, I.M., Puertas, M.L.: On the metric dimension of infinite graphs. Discrete Appl. Math. 160(18), 2618-2626 (2012)

5. Cáceres, J., Hernando, C., Mora, M., Pelayo, I.M., Puertas, M.L., Seara, C., Wood, D.R.: On the metric dimension of cartesian products of graphs. SIAM J. Discrete Math. 21, 423-441 (2007)

6. Chartrand, G., Eroh, L., Johnson, M.A., Oellermann, O.R.: Resolvability in graphs and the metric dimension of a graph. Discrete Appl. Math. 105, 99-113 (2000)

7. Chiba, N., Nishizeki, T., Abe, S., Ozawa, T.: A linear algorithm for embedding planar graphs using PQ-trees. J. Comput. Syst. Sci. 30, 54-76 (1985)

8. Chrobak, M., Payne, T.H.: A linear-time algorithm for drawing a planar graph on a grid. Inf. Process. Lett. 54, 241-246 (1995)

9. Díaz, J., Pottonen, O., Serna, M., van Leeuwen, E.J.: Complexity of metric dimension on planar graphs. J. Comput. Syst. Sci. 83, 132-158 (2017)

10. Dudenko, M., Oliynyk, B.: On unicyclic graphs of metric dimension 2. Algebra Discrete Math. 23, 216-222 (2017)

11. Epstein, L., Levin, A., Woeginger, G.J.: The (weighted) metric dimension of graphs: hard and easy cases. Algorithmica 72, 1130-1171 (2015)

12. Fernau, H., Heggernes, P., van't Hof, P., Meister, D., Saei, R.: Computing the metric dimension for chain graphs. Inf. Process. Lett. 115, 671-676 (2015)

13. Foucaud, F., Mertzios, G.B., Naserasr, R., Parreau, A., Valicov, P.: Identification, location-domination and metric dimension on interval and permutation graphs. II. Complex. Algorithms Algorithmica 78, 914-944 (2017)

14. Foucaud, F., Mertzios, G.B., Naserasr, R., Parreau, A., Valicov, P.: Identification, location-domination and metric dimension on interval and permutation graphs. I. Bounds Theor. Comput. Sci. 668, 43-58 (2017)

15. Garijo, D., González, A., Márquez, A.: The difference between the metric dimension and the determining number of a graph. Appl. Math. Comput. 249, 487-501 (2014)

16. González, A., Hernando, C., Mora, M.: Metric-locating-dominating sets of graphs for constructing related subsets of vertices. Appl. Math. Comput. 332, 449-456 (2018)

17. Grigorious, C., Manuel, P., Miller, M., Rajan, B., Stephen, S.: On the metric dimension of circulant and Harary graphs. Appl. Math. Comput. 248, 47-54 (2014)

18. Harary, F., Melter, R.A.: On the metric dimension of a graph. Ars Comb. 2, 191-195 (1976)

19. Hernando, C., Mora, M., Pelayo, I.M., Seara, C., Wood, D.R.: Extremal graph theory for metric dimension and diameter. Electron. J. Comb. 17, R30 (2010)

20. Hernando, C., Mora, M., Pelayo, I.M.: Resolving dominating partitions in graphs. Discrete Appl. Math. 266, 237-251 (2019)

21. Héger, T., Takáts, M.: Resolving sets and semi-resolving sets in finite projective planes. Electron. J. Comb. 19(4), \#P30 (2012)

22. Kant, G.: Algorithms for drawing planar graphs, Ph. Dissertation, Dept. of Computer Science, university of Utrecht (1993)

23. Khuller, S., Raghavachari, B., Rosenfeld, A.: Landmarks in graphs. Discrete Appl. Math. 70, 217-229 (1996)

24. Mitchell, S.: Linear algorithm to recognize outerplanar and maximal outerplanar graphs. Inf. Process. Lett. 9, 229-232 (1979)

25. Muhammad, H., Siddiqui, A., Imran, M.: Computing the metric dimension of wheel related graphs. Appl. Math. Comput. 242, 624-632 (2014)

26. Quines C.J., Sun, M.: Bounds on metric dimension for families of planar graphs, arXiv:1704.04066v1 (2017)

27. Shanmukha, B., Sooryanarayana, B., Harinath, K.S.: Metric dimension of wheels. Far East J. Appl. Math. 8(3), 217-229 (2002)

28. Slater, P.J.: Leaves of trees. Congr. Numerantium 14, 549-559 (1975)

29. Slater, P.J.: Dominating and reference sets in a graph. J. Math. Phys. Sci. 22, 445-455 (1988)

30. Sudhakara, G., Hemanth Kumar, A.R.: Graphs with metric dimension two-A characterization. Int. J. Math. Comput. Sci. 3, 1128-1133 (2009)

31. Vatandoost, E., Behtoei, A., Golkhandy Pour, Y.: Cayley graphs with metric dimension two-A characterization, arXiv: 1609.06565v1 (2016) 
32. Wiegers, M.: Recognizing outerplanar graphs in linear time. In: International Workshop WG' 86 on Graph-Theoretic Concepts in Computer Science. Springer, pp. 165-176 (1987)

33. Yero, I.G., Estrada-Moreno, A., Rodrí guez-Velázquez, J.A.: Computing the k-metric dimension of graphs. Appl. Math. Comput. 300, 60-69 (2017)

Publisher's Note Springer Nature remains neutral with regard to jurisdictional claims in published maps and institutional affiliations.

\section{Affiliations}

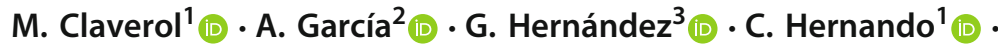

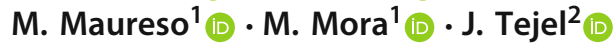

M. Claverol

merce.claverol@upc.edu
A. García
olaverri@unizar.es
G. Hernández
gregorio@fi.upm.es
C. Hernando
carmen.hernando@upc.edu
M. Maureso
montserrat.maureso@upc.edu
J. Tejel
jtejel@unizar.es
1 Departament de Matemàtiques, Universitat Politècnica de Catalunya, Barcelona, Spain
2 Departamento de Métodos Estadísticos, IUMA, Universidad de Zaragoza, Zaragoza, Spain
3 Departamento de Matemática Aplicada a las TIC, Universidad Politécnica de Madrid, Madrid, Spain 